Purdue University

Purdue e-Pubs

Open Access Theses

Theses and Dissertations

January 2015

\title{
The Application of Stereoscopic PIV in a Liquid- fueled Gas Turbine Combustor
}

Andrew Pratt

Purdue University

Follow this and additional works at: https://docs.lib.purdue.edu/open_access_theses

\section{Recommended Citation}

Pratt, Andrew, "The Application of Stereoscopic PIV in a Liquid-fueled Gas Turbine Combustor" (2015). Open Access Theses. 1158. https://docs.lib.purdue.edu/open_access_theses/1158 


\section{PURDUE UNIVERSITY \\ GRADUATE SCHOOL \\ Thesis/Dissertation Acceptance}

This is to certify that the thesis/dissertation prepared

By Andrew Pratt

Entitled

THE APPLICATION OF STEREOSCOPIC PIV IN A LIQUID-FUELED GAS TURBINE COMBUSTOR

For the degree of Master of Science in Aeronautics and Astronautics

Is approved by the final examining committee:

PROFESSOR ROBERT LUCHT

Chair

PROFESSOR CARSON SLABAUGH

PROFESSOR PAVLOS VLACHOS

To the best of my knowledge and as understood by the student in the Thesis/Dissertation Agreement, Publication Delay, and Certification Disclaimer (Graduate School Form 32), this thesis/dissertation adheres to the provisions of Purdue University's "Policy of Integrity in Research” and the use of copyright material.

Approved by Major Professor(s): Robert Lucht

Approved by: Professor William Anderson

$10 / 15 / 2015$ 
THE APPLICATION OF STEREOSCOPIC PIV

IN A LIQUID-FUELED GAS TURBINE COMBUSTOR

\author{
A Thesis \\ Submitted to the Faculty \\ of \\ Purdue University \\ by \\ Andrew Charles Pratt \\ In Partial Fulfillment of the \\ Requirements for the Degree \\ of \\ Master of Science in Aeronautics and Astronautics
}

December 2015

Purdue University

West Lafayette, Indiana 
It required an unhesitating boldness to undertake a venture so few thought could succeed, an almost exuberant enthusiasm to carry across the many obstacles and unknowns, but most of all a completely unprejudiced imagination in departing so drastically from the known way.

- J. van Lonkhuyzen, 1951, in discussing the problems faced in designing the Bell XS-1, the first supersonic airplane 
To my grandfather Richard Hill. 


\section{ACKNOWLEDGMENTS}

My most sincere gratitude is extended to Professor Lucht who took a chance on me two years ago. His support has been immeasurable.

Many thanks to my two additional committee members Professor Vlachos and Professor Slabaugh. It is without question that I would not be here today if were not for Professor Slabaughs daily mentorship (and friendship) both in and out of the lab.

The support of Scott Meyer throughout the development, integration, and operation of the high pressure test rig is greatly appreciated. Rob McGuire was also instrumental in the hardware fabrication, modification, and just making things work.

Always willing to lend a helping hand, thank you to my labmates: Rohan Gejji, Bob Zhang, Nicole Vaughn, Mike Bedard, and Brandon Kan.

Finally, thank you to my family who has always supported me to pursue my dreams. Specifically, I would like to recognize my fianc, Frances, who has the unfortunate task of putting up with me on a daily basis. 


\section{PREFACE}

This document describes the application and validation of $\mathrm{kHz}$ SPIV measurements collected in a high thermal power liquid-fueled gas turbine combustor. Chapter one discusses the motivation and background of the research. Chapter two provides an introduction to particle image velocimetry as a diagnostic tool in reacting flows. Chapter three details the facility and experimental methods employed in this research. Chapter four provides a comparison of the stereoscopic PIV measurements to that of 2-component PIV measurements that were collected simultaneously. Chapter five summarizes these efforts and provides a projection for future directions that can be built from them. 


\section{TABLE OF CONTENTS}

LIST OF TABLES ................................... viii

LIST OF FIGURES ........................... ix

ABSTRACT ............................... xi

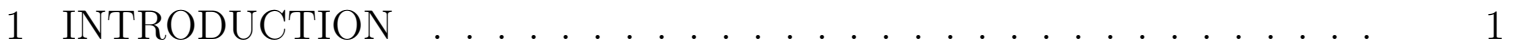

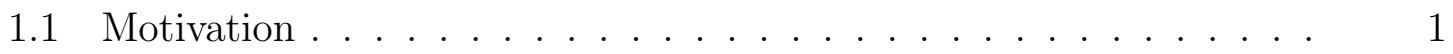

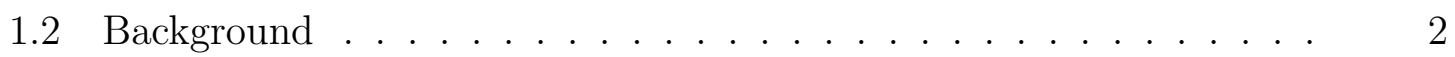

1.3 Objectives ................................. 4

2 PARTICLE IMAGE VELOCIMETRY . . . . . . . . . . . . . . . . . . 5

2.1 Seeding . . . . . . . . . . . . . . . . . . . . . 7

2.2 Illumination and Imaging . . . . . . . . . . . . . . . . . . . . . 9

2.3 Image Analysis . . . . . . . . . . . . . . . . . . . . . . 10

2.3.1 Image Pre-processing . . . . . . . . . . . . . . . . . . . . . 11

2.3.2 PIV Cross-Correlation . . . . . . . . . . . . . . 13

2.3.3 Vector validation and post processing . . . . . . . . . 15

2.4 Stereoscopic PIV . . . . . . . . . . . . . . . . . . . . . . . . . 16

2.4.1 Image Mapping . . . . . . . . . . . . . . . . . . . . . . . 17

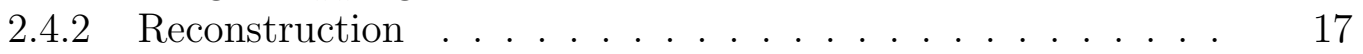

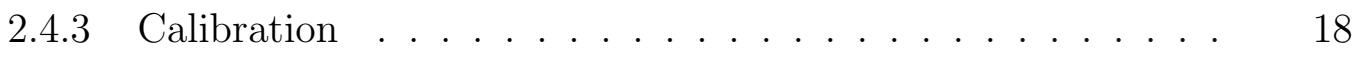

2.4.4 Self-Calibration ................... 18

3 EXPERIMENTAL FACILITY AND CONFIGURATION . . . . . . . . . 21

3.1 Facility and Test Stand . . . . . . . . . . . . . . . . . . . 22

3.2 Experiment Configuration . . . . . . . . . . . . . 23

3.2.1 Test Article . . . . . . . . . . . . . . . . . . . . . 24

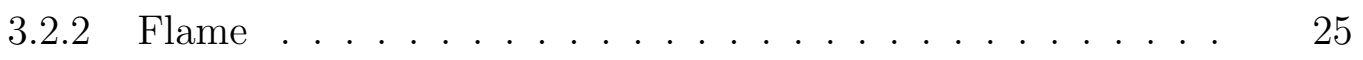

3.3 Diagnostic Configuration . . . . . . . . . . . . . 26

3.3.1 Illumination and Optics . . . . . . . . . . . . . . . . 27

3.3.2 Particle Seeder . . . . . . . . . . . . . . . . . . . . 28

3.3.3 Stereoscopic Particle Image Velocimetry . . . . . . . . . . . 28

3.3.4 2-D Particle Image Velocimetry . . . . . . . . . . . . . . . . 29

4 RESULTS AND DISCUSSION . . . . . . . . . . . . . . . . . . . . . . . . 30

4.1 Mean Comparison . . . . . . . . . . . . . . . . . . . . . 32

4.2 Time Series comparison . . . . . . . . . . . . . . . . . . . . . 34

4.3 Point Comparison . . . . . . . . . . . . . . . 36 
4.3.1 Probability density function . . . . . . . . . . . . 36

4.3.2 Correlation strength and peak ratio . . . . . . . . . 38

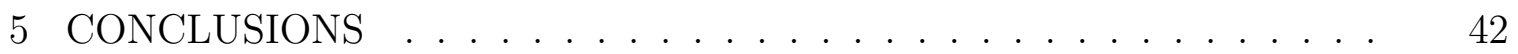

REFERENCES . . . . . . . . . . . . . . . . . . . . 43

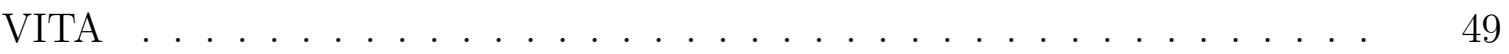




\section{LIST OF TABLES}

Table Page

3.1 Flame conditions studied . . . . . . . . . . . . . . . . . . . . 26

4.1 Correlation values and peak ratios generated at each probe location for the non-reacting case . . . . . . . . . . . . . . 40

4.2 Correlation values and peak ratios generated at each probe location for the reacting case . . . . . . . . . . . . . . . 40 


\section{LIST OF FIGURES}

Figure $\quad$ Page

1.1 NOx emissions standards from CAEP . . . . . . . . . . . . . 2

2.1 Generic PIV system configuration . . . . . . . . . . . . . . . . 10

2.2 Timing diagram . . . . . . . . . . . . . . . . . . 10

2.3 Example of liquid droplet masking . . . . . . . . . . . . . . . . . 12

2.4 Example of sliding background filter . . . . . . . . . . . . . . . 12

2.5 Example of normalization filter . . . . . . . . . . . . 13

2.6 Cross-correlation method . . . . . . . . . . . . . . . 14

2.7 Multi-pass interrogation scheme . . . . . . . . . . . . . . . 15

2.8 Basic SPIV camera configurations . . . . . . . . . . . . . . . 16

2.9 Example of image dewarping . . . . . . . . . . . . . . . 17

2.103 -component reconstruction geometry . . . . . . . . . . . . . 19

3.1 Combustor schematic . . . . . . . . . . . . . . . 25

3.2 Diagnostics schematic diagram f . . . . . . . . . . . . . . . 27

4.12 -component camera imaging error . . . . . . . . . . . . . . . . 31

4.23 -component camera imaging error . . . . . . . . . . . . . . . 32

4.3 Non-reacting mean velocity field . . . . . . . . . . . . . . . 33

4.4 Reacting mean velocity fields . . . . . . . . . . . . . . . . . 34

4.5 Non-reacting sequence of velocity fields . . . . . . . . . . . . . . 35

4.6 Reacting sequence of velocity fields . . . . . . . . . . . . . . . 36

4.7 Non-reacting case $U_{x} \mathrm{PDF} \ldots \ldots \ldots$. . . . . . . . . . . . 37

4.8 Non-reacting case $U_{y} \mathrm{PDF} \ldots \ldots \ldots \ldots$

4.9 Reacting case $U_{x} \mathrm{PDF} \ldots \ldots \ldots \ldots \ldots$

4.10 Reacting $U_{y} \mathrm{PDF} \ldots \ldots \ldots$

4.11 Correlation Maps for the non-reacting case . . . . . . . . . . . . . 41 
Figure $\quad$ Page

4.12 Correlation Maps for the Reacting case . . . . . . . . . . . . . . . . . 41 


\begin{abstract}
Pratt, Andrew Charles MSAA, Purdue University, December 2015. The application of Stereoscopic PIV in a Liquid-fueled Gas Turbine Combustor. Major Professor: Robert P. Lucht, School of Mechanical Engineering.

Strict regulations on aviation gas turbine engine emissions and fuel consumption have driven the development of new lean burning, efficient gas turbine injectors. In an effort to increase fundamental understanding and support modeling efforts, great advancements have taken place in experimental measurement techniques. Specifically, in the field of laser diagnostics.

This work describes the application of high repetition rate stereoscopic particle image velocimetry to a gas turbine combustor operating at representative engine conditions. A motivation and brief background of this research is provided. An introduction to Stereoscopic Particle Image Velocimetry (SPIV) and its development is included with a description of the experimental systems and the challenges associated with acquiring useful data in high pressure and high thermal power. The facility capabilities and test stand capabilities are presented along with the operational configuration for both the experimental and diagnostic systems. Finally, results are presented from two operating conditions, one with combustion and one without. Both 3-component SPIV and 2-component PIV data were collected simultaneously at $6 \mathrm{kHz}$. The vector fields generated from both techniques are compared both qualitatively and quantitatively.
\end{abstract}




\section{INTRODUCTION}

\subsection{Motivation}

Over the past 40 years gas turbine combustors have gone through great scrutiny to reduce emissions and increase efficiency [1]. The efforts of OEM's to reduce emissions from aircraft are driven by the standards set by the International Civil Aviation Organization's (ICAO) Committee on Aviation Environmental Protection (CAEP). These standards put a cap on the amount of oxides of nitrogen (NOx), carbon monoxide (CO), and unburnt hydrocarbons (UHC) that can be emitted over a Landing and Take-Off (LTO) cycle. This cycle covers the take-off, climb, descent, and taxi/ground idle portions of the aircraft's operation [2]. The predicted growth of aviation of approximately $5 \%$ annually. for the next 25 years with a four-fold increase in aviation fuel consumption by 2050 compared to 1992 has exerted immense pressure on gas turbine emissions [3].

The Intergovernmental Panel for Climate Change (IPCC), has placed a major focus on $\mathrm{CO}_{2}$ and $\mathrm{NOx}$ emissions. During combustion, the operating conditions that promote reduction in $\mathrm{CO}_{2}$ emissions, increase $\mathrm{NOx}$ emissions and vice versa. A tradeoff in operating conditions to keep both under emission norms is important. For aviation gas turbines, from a local air quality as well as global climate perspective, a significant focus has been placed on NOx reduction technologies. NOx, which mainly comprises of $\mathrm{NO}$ and $\mathrm{NO}_{2}$, is a participant in ozone formation and contributes to formation of nitric acid and acidification of aerosols and rain [4]. A summary of gas turbine engines and their NOx emissions compared to CAEP levels are shown in Figure 1.1.

The primary NOx in combustion systems is nitric oxide (NO) which is formed through combustion mechanisms. Thermal NO production is sensitive to temperature 


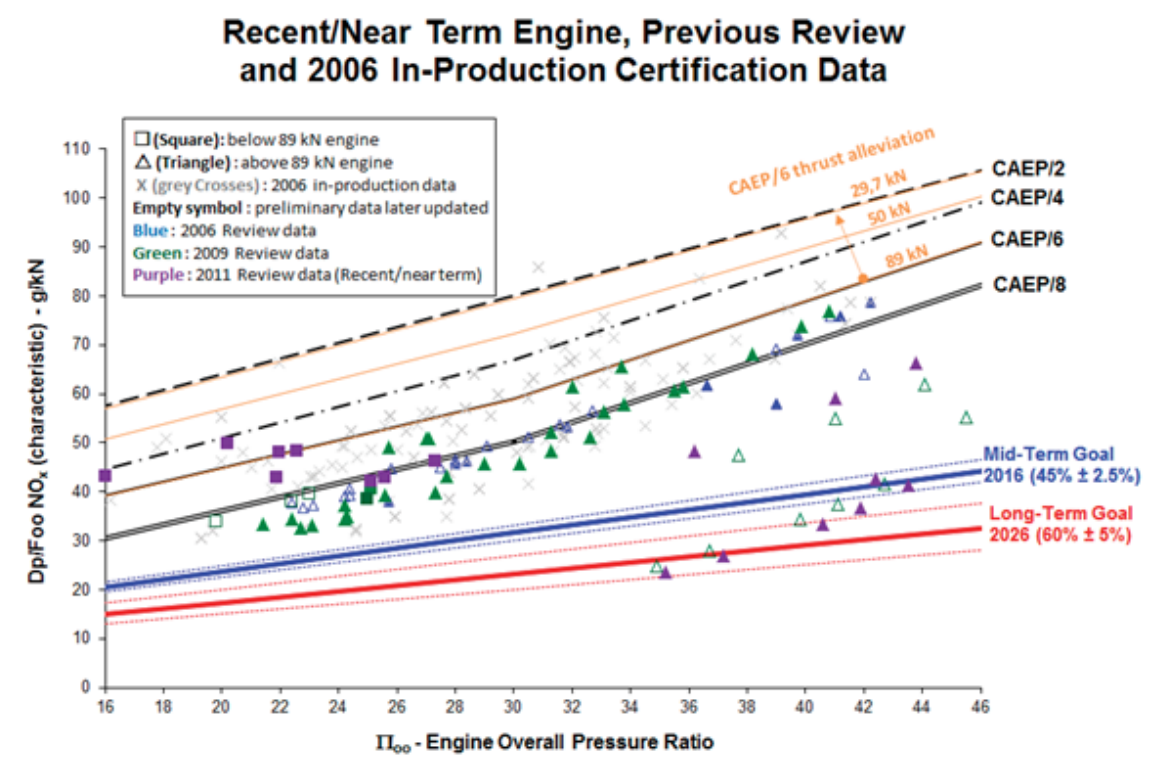

Figure 1.1: NOx emission standards from CAEP/2 through CAEP/8 along with mid-term and long-term goals [3].

and is highest at temperatures in excess of $1800 \mathrm{~K}[5,6]$. All low dry NOx technologies are hence aimed at operating at overall low equivalence ratios, thus reducing, both temporally and spatially the existence of stoichiometric, high temperature zones in the combustor. The success of low NOx gas turbine injector technology has a critical dependence on the fuel-air vaporizing-mixing and the flame holding strategies. With much of the gas turbine industry working on these lean combustion technologies, it is important that experimental tools are developed to rapidly and effectively evaluate the operation of gas turbine injectors at realistic engine conditions. The current work focuses on the application of a non-intrusive velocity field measurement diagnostic, known as Stereoscopic Particle Image Velocimetry (SPIV), in a windowed combustor capable of replicating modern gas turbine combustor operating conditions.

\subsection{Background}

Laser-based diagnostics provide a non-intrusive means of collecting high-fidelity measurements of flow properties. Provided sufficient optical access these techniques 
have no effect on the flow which is being interrogated. This is in stark contrast to standard physical probes such as thermocouples and hot wires anemometers whose physical presence in the flow can significantly effect the measured quantities. In addition to this, laser diagnostics are not limited by the temperature of the flow field. This makes them excellent candidates for use in combustion research.

Even with the advantage of being non-intrusive, the application of laser-based techniques in modern combustors is quite challenging. Probably the biggest and most obvious challenge, is maintaining optical access of the combustor. This requires extensive design efforts to ensure that the windows will withstand the extremely high temperatures and pressures, which can be greater than $2000 \mathrm{~K}$ and 50 bar, respectively. Only a select few research groups in the world have achieved these testing capabilities. This is due to the high cost associated with large-scale experiments in addition to the physical challenges of performing useful measurements in such environments [7-16].

With that in mind the recent commercial availability of high-repetition-rate (on the order of 5 - 10 kilohertz), diode-pumped solid-state (DPSS) lasers with short pulse durations (on the order of $10 \mathrm{~ns}$ ) has made it possible to acquire temporallyresolved planar measurements of velocities and scalars in reacting flows [17-19]. In the past, researchers have relied on stochastic, or (at best) phase-locked measurements to study reacting flows. With the ability to record at these high repetition rates the scientific community has gained a much improved understanding of turbulent reacting flow [20-33]. The temporally and spatially resolved data that can now be collected, holds promise for the future of combustion research.

One technique that is of specific interest is particle image velocimetry. Particle Image Velocimetry (PIV) is a very powerful technique in which successive images of elastically scattered laser light from seed particles in a flow are used to measure the velocity field. As is the case of a windowed combustor, enclosed test sections present challenges for this technique due to reflections and scattered light from internal surfaces. Being at the same wavelength as the signal, it is not possible to optically 
filter these noise sources from the particle field. Seeding the flow in high pressure experiments is also a challenge due to geometric constraints and window contamination concerns. Field of view restrictions also limit the application of more advanced techniques such as stereoscopic PIV [34].

While the motivation for applying these advanced diagnostics is clear, sigificant issues remain. Despite the high average power output of a DPSS laser, the single-shot pulse energy is quite low. For comparison a similar $10 \mathrm{~Hz}$ laser has almost 2 orders of magnitude greater pulse energy. To make matters worse, the systems typically have poor beam intensity profiles and can experience significant shot-to-shot variation. Still, these techniques have displayed the potential to provide valuable information for both the computational and experimental combustion research communities.

In recent works, Slabaugh et al. [7] has documented the development and demonstration of a high power, optically-accessible combustion test rig designed to support high resolution optical measurements in a high-power swirl flame. Boxx et al. [35] and Slabaugh et al. $[34,36]$ have reported the first successful simultaneous measurements of velocity and scalar fields in mid to high-power swirl flames using kilohertz planar diagnostics. Beyond making these challenging measurements, the extraction and interpretation of quantitative flame data is of key importance. This work will discuss the results of simultaneous SPIV and 2-D PIV measurements collected in liquid-fueled flames operating at high thermal powers.

\subsection{Objectives}

The objective of this work is two-fold. Firstly, demonstrate the ability to collect SPIV measurements in a optically accessible aero-engine combustion test rig operating at realistic engine conditions with liquid fuel. Secondly, validate these stereo measurements with 2-D PIV data that are collected simultaneously. 


\section{PARTICLE IMAGE VELOCIMETRY}

In a very basic sense PIV can be described as a technique of measuring fluid flow velocity by observing the movement of tracer particles. At the core of this is the classic definition of velocity

$$
u(x, t)=\frac{\Delta x(x, t)}{\Delta t},
$$

where $u$ is the local velocity of a particle at position $x$ and time $t$ and $\Delta x$ is the displacement of said particle over time interval $\Delta t$. The ability to acquire quantitative results from Particle-Tracking Velocimetry has been around for the better part of a century [37]. A predecessor and close relative of PIV is Laser-Speckled Velocimetry which takes its roots from solid mechanics [38]. The viability of LSV in fluid flow was first demonstrated by [39-41]. In 1984, Adrian et al. [42,43] defined PIV as a "distinct mode of pulsed-light velocimetry" [44]. They argued that, in fluid applications, it is unlikely that particle density is high enough to create speckle patterns in the scattered light as is a requirement of LSV. Rather, the observer actually records images of particles. This led to the realization that much work thought to be LSV at that time was in fact PIV. From that point on PIV became the primary method by which velocity measurements in fluid flows were acquired [38,44]. In 1991 Willert [45] outlined his implementation of computational digital PIV, greatly reducing image processing time. Up to then, velocity data was extracted from photographs by optomechanical techniques. Still, there was room for improvement. The repetition rate of velocity measurements was limited by the camera frame rate (30 Hz at the time). It has not been until recent years that the development of high-repetition rate (5-10 $\mathrm{kHz}$ ), diode-pumped solid-state (DPSS) lasers with short pulse durations (on the order 
of $10 \mathrm{~ns}$ ) and high-speed cameras, have made temporally-resolved planar velocity measurements feasible $[7,17,18,34,36,46-49]$.

A key disadvantage of planar PIV is that it can only provide velocity measurements of particles within the object plane (2 velocity components). Any out of plane particle motion can not be resolved. Unfortunately, this out of plane motion is then projected onto the target plane, inducing a bias in the planar velocity measurements. To account for this error a second camera is added off-axis from the original camera, but still focused on the same object plane. This introduces two more velocity components. Approaching the situation as system of equations, there are three unknowns: the two in-plane directions of motion $(\Delta x, \Delta y)$ and the single out-of-plane direction $(\Delta z)$. If only a single camera is used only two equations are available. With the addition of the second off-axis camera, two more equations are added. Thus creating an overdetermined system, with four equations and only three unknowns. This means the third velocity component can readily be resolved [50]. With the ability to resolve all three velocity components, highly three-dimensional flows such as those created by gas turbine fuel injectors can be better characterized. This two camera method is referred to as Stereoscopic Particle Image Velocimetry and has been well documented for application in wind tunnel flows [51-56]. Multiple groups have also demonstrated SPIV capabilities in atmospheric pressure combustors [29,57-60]. Most recently threecomponent data has been collected in a high pressure gaseous fueled swirl burner at DLR, Germany [36]. When considering PIV in a liquid hydrocarbon reacting flow, there are multiple confounding physical phenomenon related to the presence of chemical reactions that must be taken into consideration [61]:

- Heat release from combustion results in a very rapid rise in fluid temperature. In a gas turbine combustor, burning et-A and air flame can reach temperatures in excess of $2000 \mathrm{~K}$. 
- Hydrocarbon fuels with high aromatic content emit high intensity broadband light as a result of the flame radiation. This flame luminosity increases the background noise, effectively reducing the signal-to-noise ratio.

- Steep gradients in thermo-fluid dynamic properties such as temperature and density cause dynamic range issues near the reaction front where properties can change orders of magnitude over a very short distance $(<1 \mathrm{~mm})$.

- Fluctuations in the fluid mixture composition and density throughout the fluid volume produce an inhomogeneous field of index of refraction. Resulting in defocussing of particles in the object plane.

- In the particular case of liquid-fuel combustion, light scattered off fuel droplets can interfere with the effective tracking of actual seed particles; wherein fuel droplets are constantly changing in size and cannot be assumed to track the flow.

The following sections will discuss the key aspects of PIV and how the aforementioned issues can be addressed to mitigate their negative effects on the quality of data collected.

\section{$2.1 \quad$ Seeding}

In order to acquire accurate flow velocity measurements, careful consideration must be given to the material selection and sizing of the seeding particles. As an indirect measurement, PIV relies on the assumption that the flow tracers perfectly follow the flow. In addition to this assumption the particles must effectively scatter light to be recorded clearly. Finally, the seed density must be sufficiently high to mark enough points in the flow field for spatial resolution. The selection of particles requires the researcher to carefully assess the various properties of the particles, the 
fluid, and the flow [38]. For particle sizing, the key parameter is the particle time constant, $\tau_{p}$, determined by

$$
\tau_{p}=\frac{\left(\rho_{p}-\rho_{f}\right) d_{p}^{2}}{18 \rho_{f} v_{f} \phi}
$$

where $\rho_{p}$ is the particle density, $\rho_{f}$ the fluid density, $d_{p}$ the particle diameter, $v_{f}$ the fluid kinematic viscosity, and $\phi$ is a function to account for particle drag depending on the particle Reynolds number, $R e_{p}$, as seen in equation 2.3 [38].

$$
\phi= \begin{cases}1+\frac{3}{16} R e_{p}, & R e_{p} \leq 0.01 \\ 1+0.131 R e_{p}^{0.82-0.05 \log _{10} R e_{p}}, & 0.01 \leq R e_{p} \leq 20 \\ 1+0.1935 R e_{p}^{0.6305}, & 20 \leq R e_{p} \leq 260\end{cases}
$$

The flow tracers must be sized such that the particle time constant is significantly less than that of the time scales of the flow being studied [38,62]. This is typically represented by the Stoke's number defined by the product of the particle time constant and fluid velocity, divided by the diameter of the particle. The Stoke's number should be much less than 1 in order to be consider to accurately track the flow. With particle sizing accounted for, the proper material must be selected to withstand the extreme temperatures found in a flame. [62] Provides a review of many different seeding particles and their thermal limitations. Common particle choices for reacting flows include $\mathrm{Al}_{2} \mathrm{O}_{3}$ [63-66], $\mathrm{TiO}_{2}[34,67,68]$, and $\mathrm{ZrO}_{2}[68,69]$. LaVision Inc. offers a surface treated $\mathrm{TiO}_{2}$ seeding material designed specifically for use in combustion experiments and is capable of withstanding temperatures greater than $2000 \mathrm{~K}$. In deciding between the thermally capable materials, it is often of use to consider the light scattering behavior of the different options. Along with the shape and size of the particle, the refractive index of the material will determine whether or not the 
particle will scatter a sufficient amount of light to be collected by the optical recording device [70].

\subsection{Illumination and Imaging}

Illumination of the object plane is typically provided by a pulsed laser system with short pulse durations (on the order of $10 \mathrm{~ns}$ ). Solid-state Nd:YAG lasers emitting 532 nm light are a common choice due to their commercial availability. The laser light is manipulated into a thin "sheet" by a series of cylindrical optics and passed through the seeded flow field. The elastically scattered light from the particle field is then focused onto the video chip of a camera such as a Complementary Metal Oxide Semiconductor (CMOS) array. CMOS cameras have a rectangular array of pixel sensors that convert the exposed light energy into voltages. The intensity of the signal is proportional to the light energy that hits each pixel during the exposure time (duration of time light is entering the sensor array). For PIV applications the exposure time is set by the laser pulse duration as it is much shorter than the camera's shutter exposure. However, this discrepancy between the camera exposure and the laser pulse duration can be troublesome when the flow is combusting. As mentioned earlier, the flame luminosity of a hydrocarbon flame can be very intense. Since the camera's exposure is so much longer than the laser pulse, significant noise can be added to the images due to the luminosity of the flame. In order to mitigate this effect, a narrow band filter of $5 \mathrm{~nm}$ FWHM (or less) centered on the wavelength of the laser light is installed in front of the optical lens. Figure 2.1 is a generic representation of a standard PIV system.

Normally when using CMOS cameras, images are recorded in a double-frame, single pulse mode. This means each camera exposure records the light scattered from a single laser pulse. When recording at $\mathrm{kHz}$ repetition rates, a technique called frame-

straddling is used. A timing diagram for a PIV system operating in frame straddling 


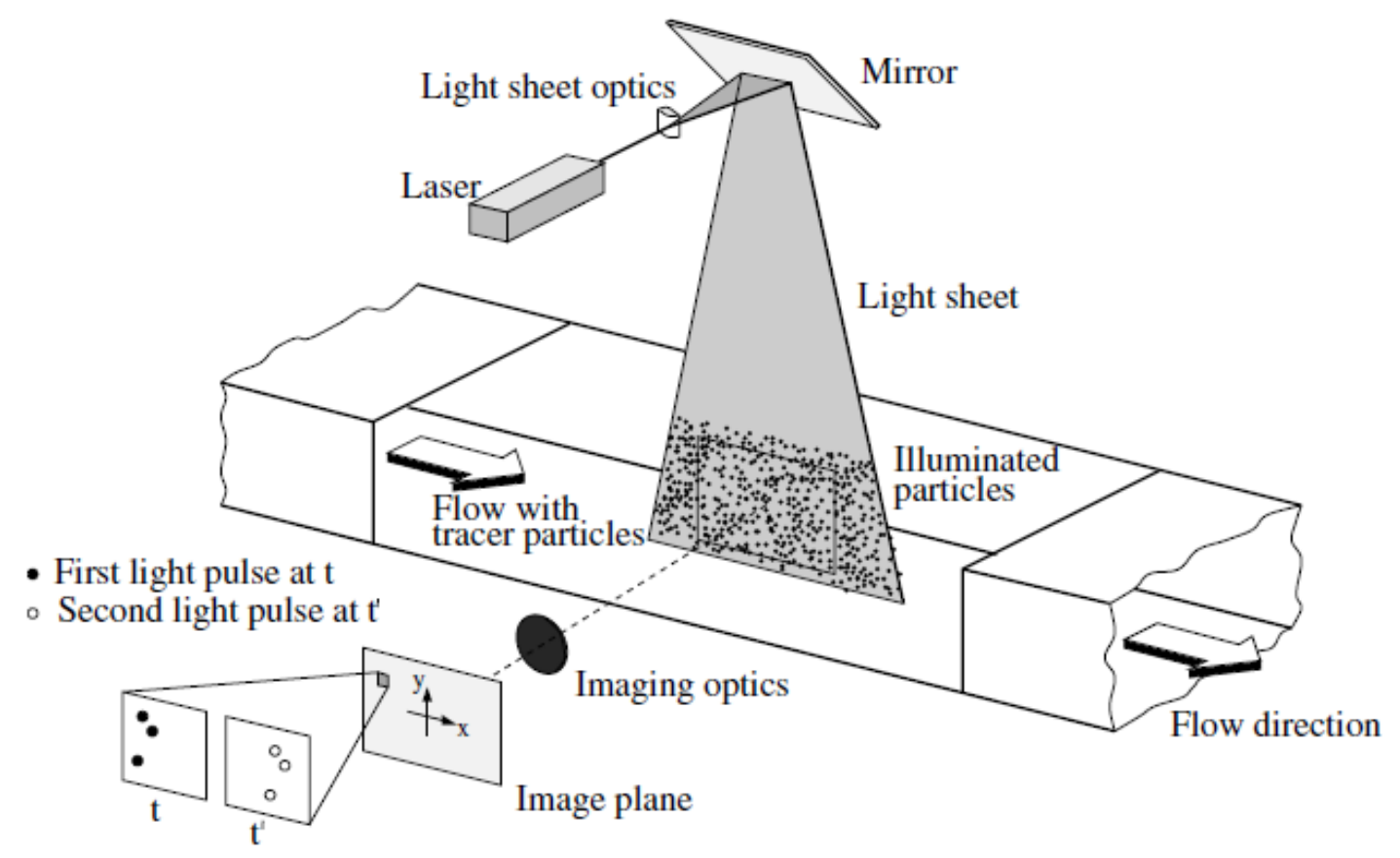

Figure 2.1: Schematic of PIV system configuration [70]

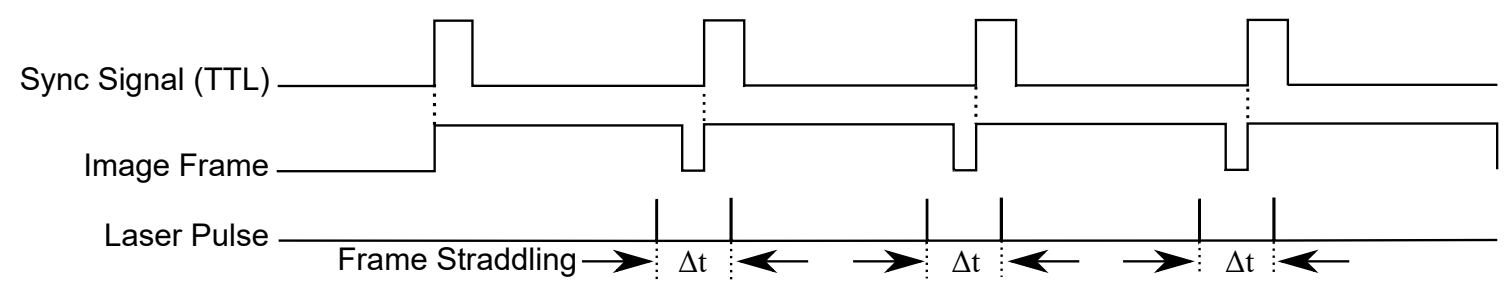

Figure 2.2: Timing diagram for frame straddling mode

mode is shown in figure 2.2. The laser pulse separation, $\Delta t$ when referring to equation 2.1, is limited by the camera shutter speed and the overall PIV system repetition rate.

\subsection{Image Analysis}

Image analysis and vector generation can be accomplished through a variety of commercially available softwares such as Insight by TSI, Flowmap by Dantec, and DaVis by LaVision. These are powerful computational tools that tend to be a "black box" to the end user. Whenever possible it is important for the researcher to take the 
time and understand the operations being carried out by the software. This section will give an overview of the general processes used to extract vector fields from the raw images collected during an experiment.

\subsubsection{Image Pre-processing}

Ideally, raw images will have a zero intensity black background with a field of bright high intensity particles overlaid. In reality this is never the case. The background contains noise caused by reflections from hardware or optics, thermal effects, and flame luminosity (in the case of reacting flows). Non-uniformities in the laser light source can be exacerbated when formed into a sheet resulting in areas of the object plane where very little light is scattered from the particles. For reacting flows, the variation in density results in inhomogeneity in the index of refraction across the object plane causing some particles to appear out of focus. Additionally, liquid fuel droplets will scatter the laser light. Since the size and shape of the liquid droplets is unknown, it cannot be assumed that the droplets are accurately tracking the flow field. An algorithmic mask must be applied to remove regions of the image where the particle size is too large. Figure 2.3 shows an example of the droplet masking applied in this work.

Other image pre-processing steps may include a geometric mask, intensity normalization, or background subtraction. A geometric mask is commonly used to remove areas of the image that are not part of the seeded scattering field. For example, if part of the test article is visible in the image, a mask should be applied over this region. In the case of minor background reflections or intensity fluctuations, a sliding background filter can be used. This works similar to a high pass filter, resulting in a uniform background intensity as seen in figure 2.4 [71].

Another way to address high intensity fluctuations is applying a particle intensity normalization. This is especially useful in the case of reacting flows where the fluctuations in refractive index of the fluid cause particle defocussing and not all par- 

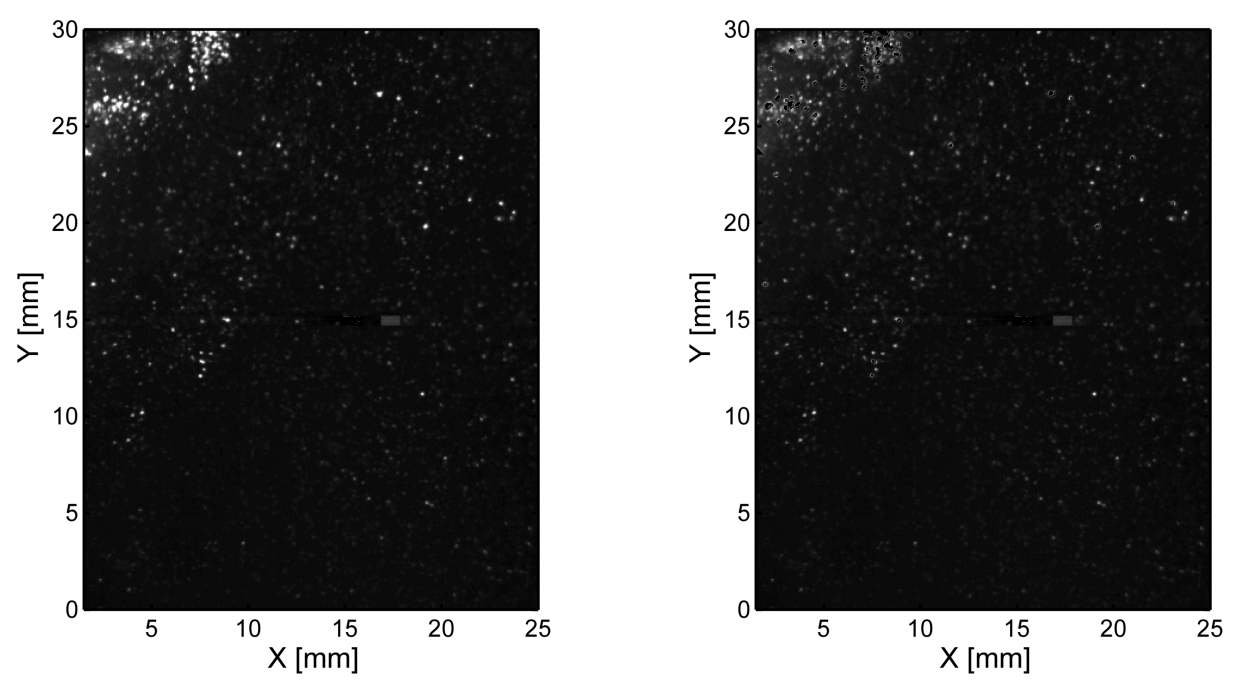

Figure 2.3: Example of a particle field in a liquid fueled reacting flow before (left) and after (right) droplet masking

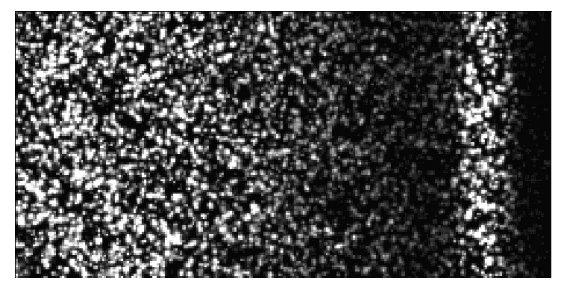

Raw image

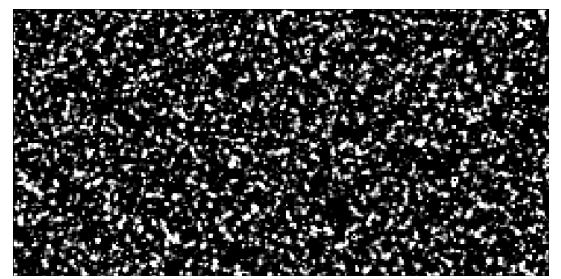

Post background filtering

Figure 2.4: Example of sliding background filter [71]

ticles have equal intensities. The filter works by sliding a window of a specified scale length over the image and extracting a local minimum. This local minimum is then subtracted from the original image, call this $\operatorname{Im} 00$. Then a window is slid over the original image again, only this time calculating a local maximum, Im01. Finally a global maximum is found for the entire original image, Im02. Im02 is divided by $\operatorname{Im} 01$ to get $\operatorname{Im} 03$. This ratio, Im03, is then multiplied by Im00, the result from local minimum subtraction. This results in a uniform particle intensity field shown in figure 2.5 [71]. 


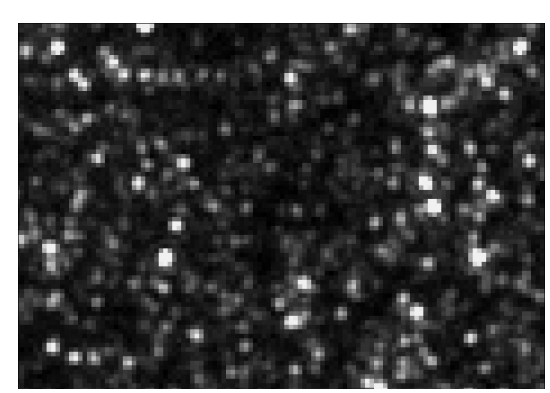

Raw image

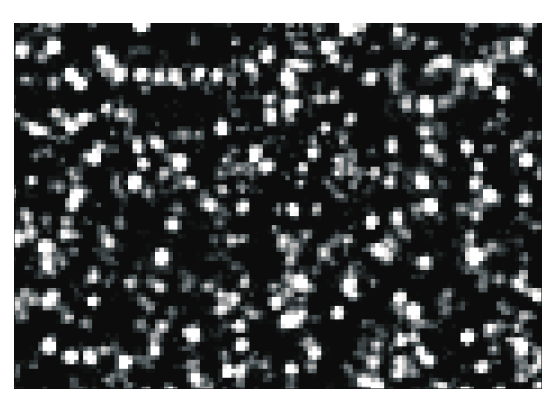

Post normalization

Figure 2.5: Example of particle intensity normalization filter [71]

\subsubsection{PIV Cross-Correlation}

The displacement of particles is determined using a spatial cross-correlation algorithm. Generally defined as

$$
R(s)=\int \tau_{1}(X) \tau_{2}(X+s) d X
$$

The image patterns $\tau_{1}(X)$ and $\tau_{2}(X)$ are divided into small regions referred to as interrogation windows. The light intensities $I\left(X, t_{1}\right)$ and $I\left(X, t_{2}\right)$ found within the interrogation windows $W_{1}\left(X-X_{I 1}\right)$ and $W_{2}\left(X-X_{I 2}\right)$ are assumed directly proportional to the recorded signals $\tau_{1}(X)$ and $\tau_{2}(X)$ such that,

$$
\begin{gathered}
\tau_{1}(X)=W_{1}\left(X-X_{I 1}\right) I\left(X, t_{1}\right) \\
\tau_{2}(X)=W_{2}\left(X-X_{I 2}\right) I\left(X, t_{2}\right),
\end{gathered}
$$

where $t_{2}-t_{1}=\Delta t$, the laser pulse separation, and $X_{I 1}$ and $X_{I 2}$ are the locations of the interrogation windows $W_{1}$ and $W_{2}$ respectively [38]. A map is then created of 


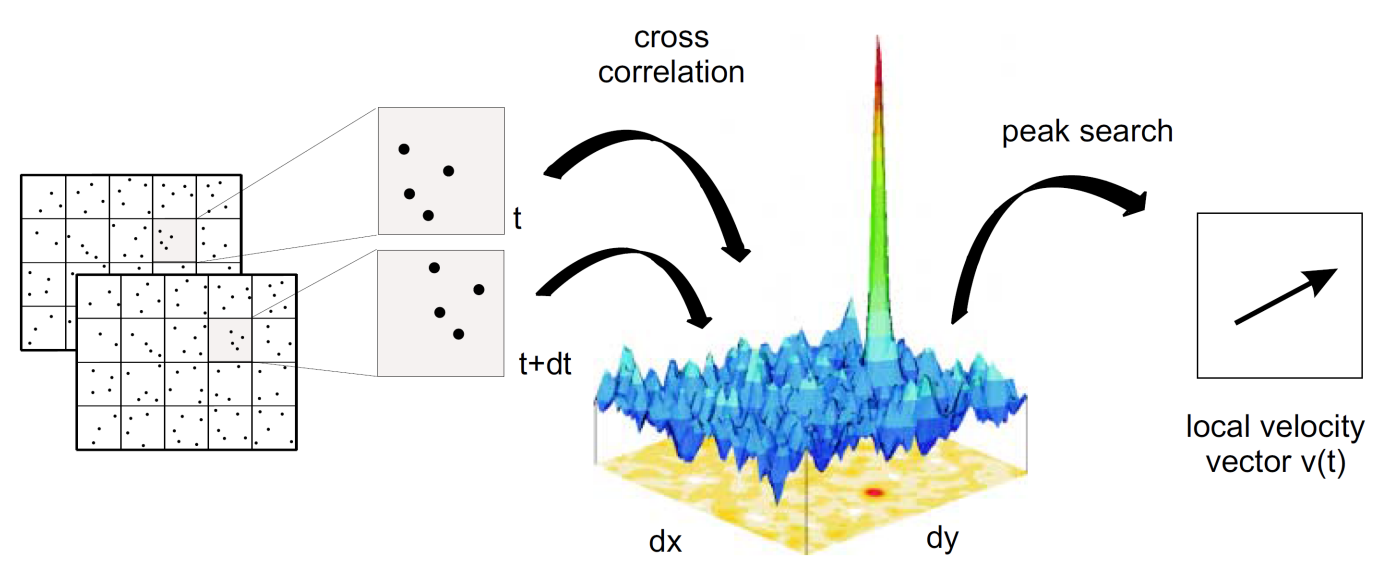

Figure 2.6: Evaluation of PIV recordings using Cross0Correlation [71]

the correlation values within each window. The location of the highest peak indicates the most probable particle displacement. This cross correlation process is shown graphically in figure 2.6.

In order to improve the accuracy of the cross-correlation a few extra methods are applied. Firstly, a multi-pass interrogation utilizes the particle image shift from the first pass to provide a reference vector field. Using this information on the second pass, the interrogation window is offset with respect to the particle image shift as seen in figure 2.7.

This ensures the correct particles are correlated. This technique is especially useful when large displacements are present. To improve spatial resolution and reduce the amount of erroneous vectors, the window size can be iteratively reduced simultaneously with the multi-pass interrogation. In regions of large velocity gradients (shear layers), the correlation signal-to-noise ratio decreases due to the broadening of the correlation peak. To correct for this the window shape is iteratively deformed so particle pairs within each window have similar displacements. This will increase the correlation strength and narrow the peak, increasing the signal-to-noise ratio $[38,70,71]$. 


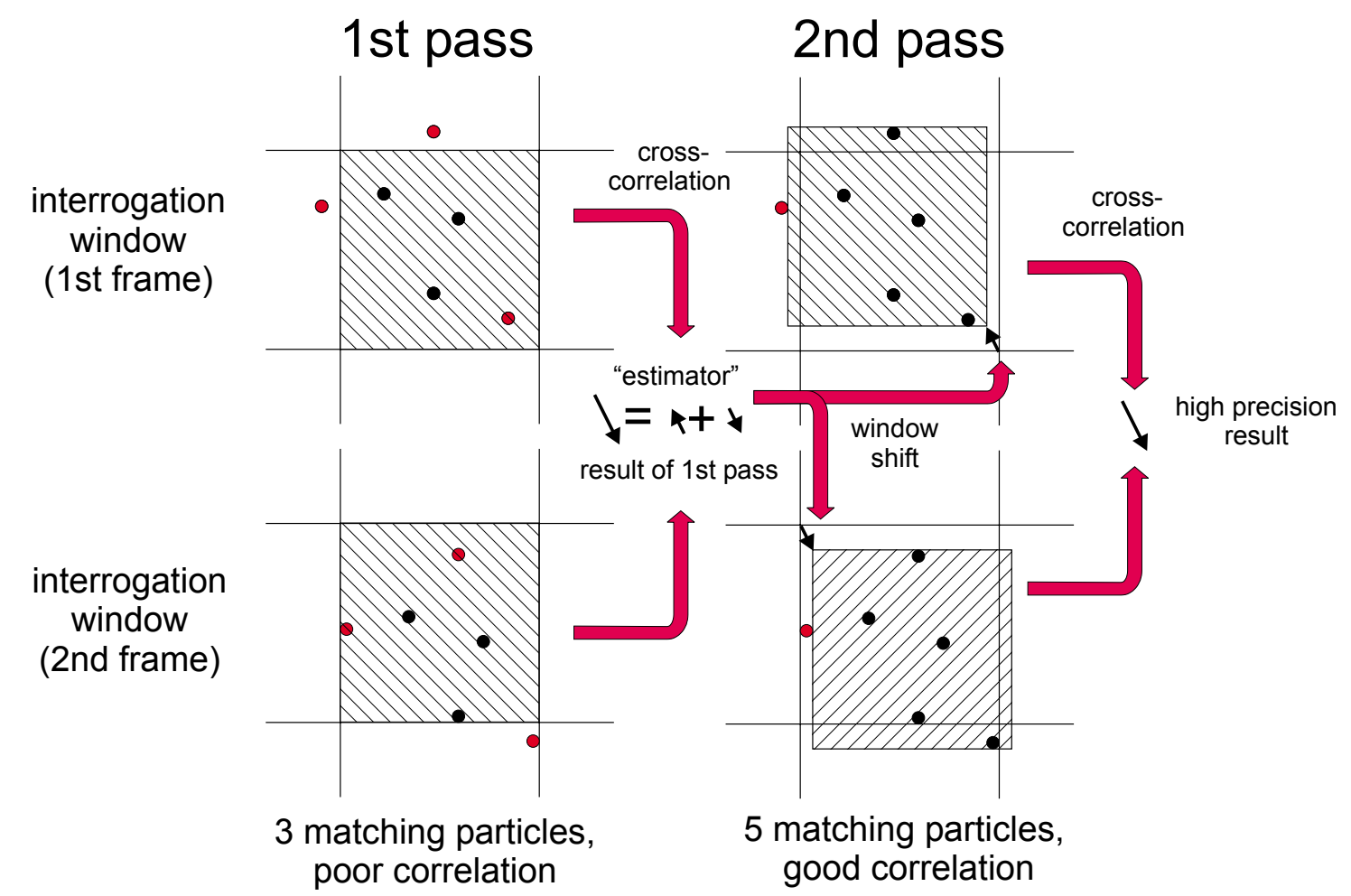

Figure 2.7: Principle of multi-pass scheme with constant interrogation window size [71]

\subsubsection{Vector validation and post processing}

Two factors that are commonly used to validate vector choices are peak ratio, $\mathrm{Q}$, and correlation value. As mentioned earlier the correlation value is the normalized peak height of the tallest peak in the interrogation window, a single correlation strength may also be given for the entire window as a result of the sum-of-correlations within the window. The peak ratio is defined as the highest correlation peak divided by the second highest correlation peak within the window. In non-reacting flows, Qfactors range from 3-4 and correlation values 0.7-0.8. As will be shown in this work, the effects of combustion on the flow field cause a reduction on both factors. Additionally, a median filter can be applied to remove any spurious vectors. A median filter compares vectors with the calculated median vector of its surrounding vectors, $+/$ - their standard deviation. If the center vector is outside the allowable range for 
both the $x$ and $y$ components, it is rejected. A median filter can be applied numerous times. Removal of these invalid vectors can create empty spaces in the vector field. If desired these empty spaces can be filled via interpolation using neighboring vectors. Finally, smoothing or denoising filters can be applied to reduce noise in the vector field.

\subsection{Stereoscopic PIV}

Figure 2.8 shows the two major types of SPIV configurations. The translational approach is limited by the angular aperture of the lenses and the significant decrease in resolution and contrast towards the edges of the field of view [51]. The angular approach is a much preferred method allowing the principle lens axis to intersect with the center of the field of view. However, since PIV calls for small depth of field lenses to reduce background noise, the entirety of the image will not be in focus on the sensor. Thus, the lens plane needs to be titled according to the Scheimpflug criterion, which states that the image plane, lens plane, and object plane must intersect in a common line as seen in the angular PIV Schematic in figure 2.8. This focusing correction does not come without side effects. The tilting of the backplane introduces perspective distortion which means the magnification factor varies across the field of view. To address this a specific calibration method is used. [51]
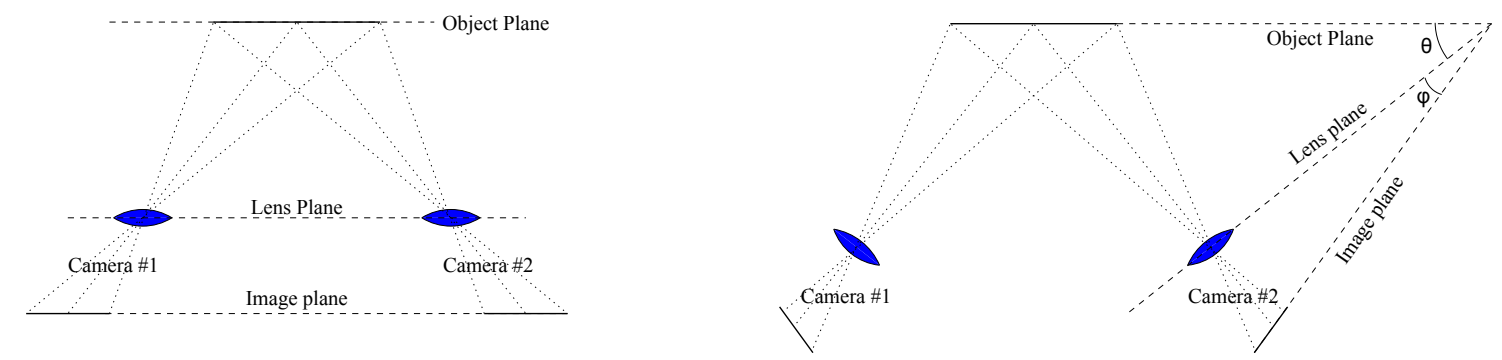

Figure 2.8: Schematics for SPIV camera configuration: Translational (left) and Angular (right) [51] 


\subsubsection{Image Mapping}

In order to properly reconstruct the three-component displacement vectors, the perspective error resulting from the Scheimpflug criterion must be corrected. This process is known as image dewarping. This is shown visually in figure 2.9. This process is typically built into the calibration portion of commercial software.

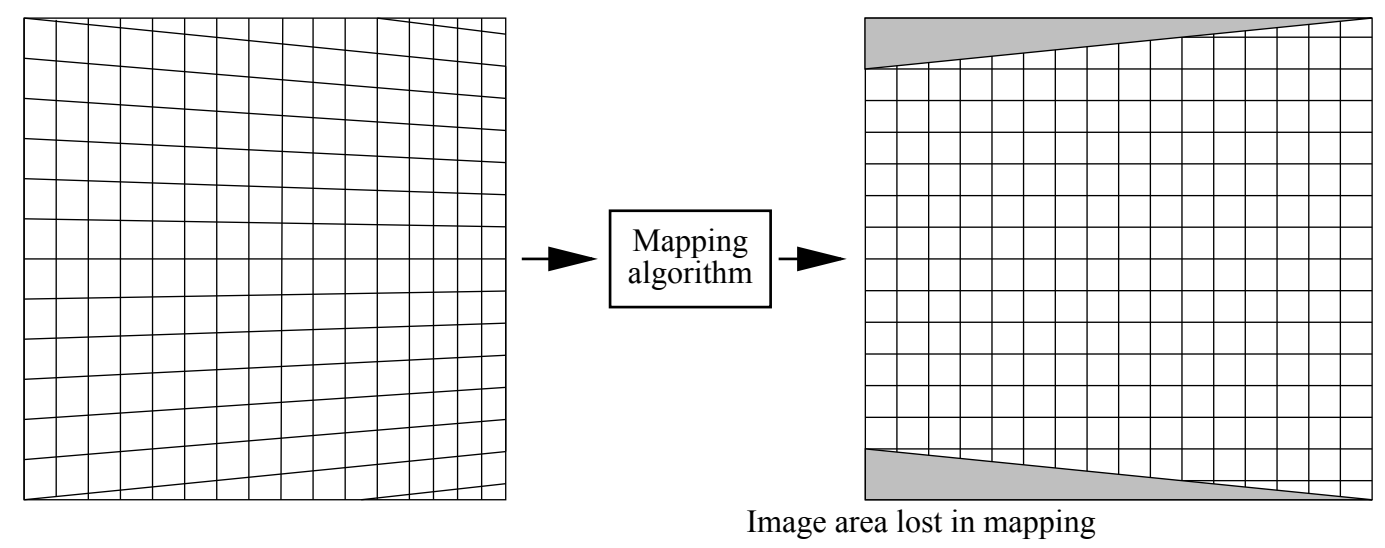

Figure 2.9: Example of image dewarping effects [70]

\subsubsection{Reconstruction}

Using basic geometry, the three velocity components can be reconstructed using equations 2.7 - 2.9 in conjunction with figure 2.10 provided by Willert [51]. In an effort to simplify the concept, a pinhole camera model is used in the geometric representations in figure 2.10. O, is a point of reference viewable by both cameras, with coordinates $\left(x_{O}, y_{O}, z_{O}\right)$ and point $\mathrm{P}\left(x_{P}, y_{P}, z_{P}\right)$ is a location where the displacement vectors are to be calculated. The light sheet is aligned with the $x$ - and $y$ - axes. The 
measured displacements corresponding viewing angles for camera 1 are given as $\left(d x_{1}\right.$, $d y_{1}$ and $\left.\alpha_{1}, \beta_{1}\right)$ respectively.

$$
\begin{aligned}
& d x=\frac{d x_{2} \tan \alpha_{1}-d x_{2} \tan \alpha_{2}}{\tan \alpha_{1}-\tan \alpha_{2}} \\
& d y=\frac{d y_{2} \tan \beta_{1}-d y_{2} \tan \beta_{2}}{\tan \beta_{1}-\tan \beta_{2}} \\
& d z=\frac{d x_{2}-d x_{1}}{\tan \alpha_{1}-\tan \alpha_{2}}=\frac{d y_{2}-d y_{1}}{\tan \beta_{1}-\tan \beta_{2}}
\end{aligned}
$$

\subsubsection{Calibration}

As mentioned earlier, a specific calibration process is required to effectively reconstruct and dewarp the stereo images. A calibration plate or dot target with a dot pattern located at two known surface levels is used as a reference to determine the exact viewing angles and magnification factors across the field of view. In the case of commercial software, most of the calibration process is automated and follow a pinhole camera model. Dots must be specified on the calibration plate to mark the correct surface level and axis orientation from which the dewarping and reconstruction process can begin.

\subsubsection{Self-Calibration}

Even when great care is taken to acquire calibration images with a dot target, small misalignments, such as an offset or rotation of the calibration plate in the measurement plane can cause significant errors in the calibration and thus, the vector 

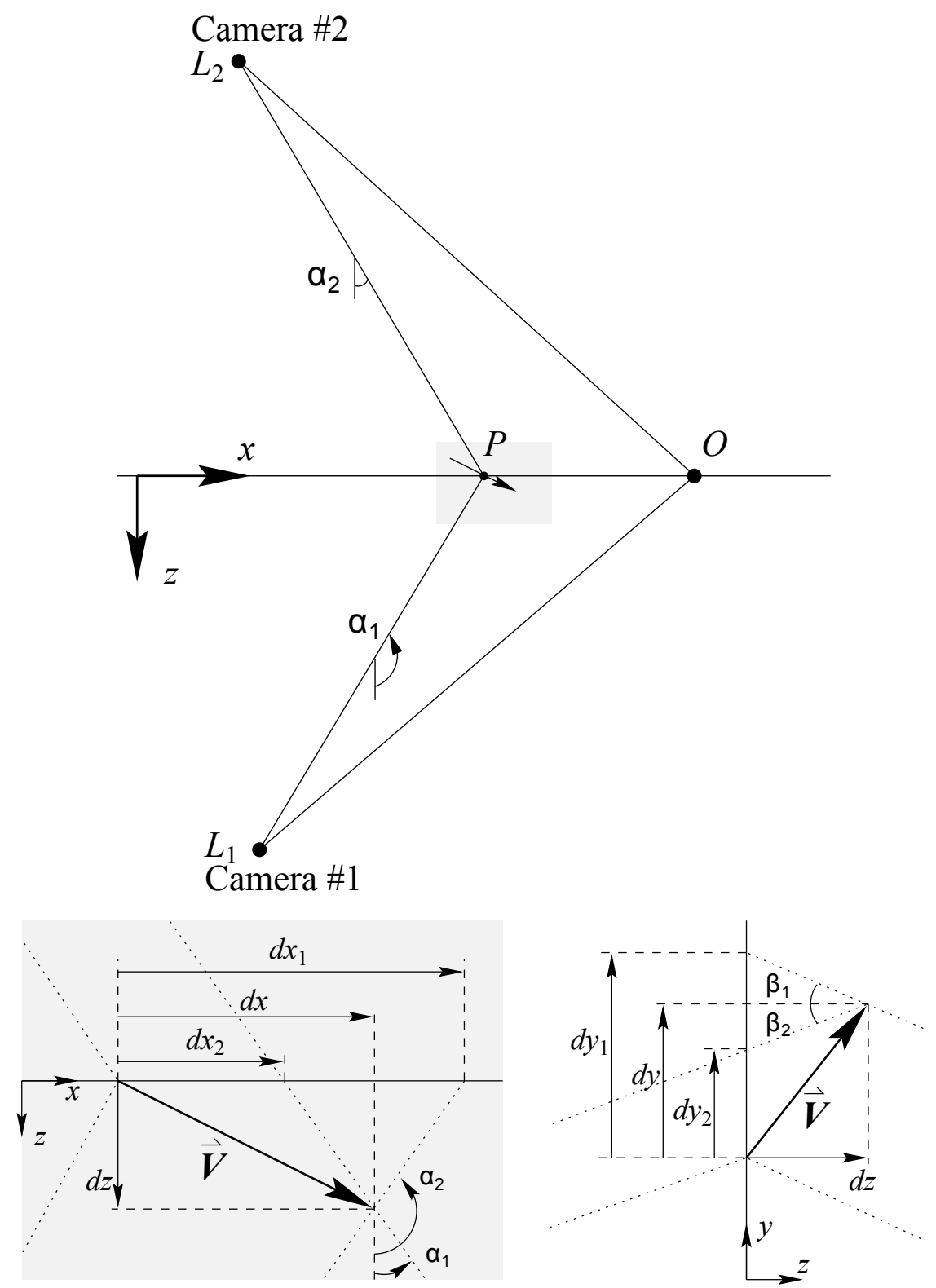

Figure 2.10: Geometric representations for reconstruction of 3-component displacement vectors [51]

generation. Fortunately, this can be accounted for in post-test analysis. Using images recorded during testing, a cross-correlation of two images acquired at the same time from each camera is calculated. The result is the disparity vector. If the images are perfectly calibrated, the disparity vectors will be zero. Adjustments are made to the 
image dewarping algorithms to eventually converge the disparity vectors across the field of view to zero. 


\section{EXPERIMENTAL FACILITY AND CONFIGURATION}

Optical measurements of combustion processes provide understanding of fundamental reacting flow physics. Development of these advanced techniques is typically performed on well-characterized, laboratory-scale burners operating at low pressure and thermal power ( $<1$ bar and $<50 \mathrm{~kW}$, respectively). Application to the study of practical combustors demands significant infrastructure development and laboratory resources. Such measurements are sought for the validation of numerical models, improving predictive capability in the areas of turbulent combustion, pollutant formation, and thermoacoustic instability.

Research facilities capable of making these measurements, while operating under well-defined operating/boundary conditions, are sparse due to the high costs and expertise required. The DLR Institute of Combustion Technology, in Stuttgart, Germany has developed a high power test stand, capable of operation at thermal powers up to $2 \mathrm{MW}$ and 40 bar maximum operating pressures. [72] This platform has been used for a multitude of advanced techniques including CARS, PLIF, and PIV. At the DLR laboratories in Köln, Germany an optically accessible high-pressure test rig and combustion chamber were designed. Similar Measurements have been taken at 6 bar with $700 \mathrm{~K}$ inlet air. The DLR test rigs all make use of a pressure vessel design, where a combustor window assembly is installed into an larger, windowed pressure housing. This design allows the combustor windows to accept thermal stresses and the pressure casing windows to handle pressure stresses [73,74].

In the United States, the NASA Glenn Research Center (GRC) in Cleveland, $\mathrm{OH}$, U.S.A. has two facilities for high-pressure combustion tests: the CE-5 and the Advanced Subsonic Combustion Rig (ASCR). Flame imaging, flow visualization and other more advanced diagnostics have been performed in each. Both experiments are supplied with non-vitiated, preheated air at temperatures from $450 \mathrm{~K}$ to $866 \mathrm{~K}$. The 
CE-5 facility can be operated with up to $4.5 \frac{\mathrm{kg}}{\mathrm{s}}$ of incoming air at pressures greater than 20 bar for sustained optical measurements in two separate test sections. One of the rigs, used for fuel injector performance characterization, can support up to $1.36 \frac{\mathrm{kg}}{\mathrm{s}}$ of inlet air and pressures up to 20 bar. The second sector leg can be used to test the interactions between adjacent injectors with total air mass flows up to 4.1 $\frac{\mathrm{kg}}{\mathrm{s}}$ at 18 bar. The ASCR is capable of supplying air at up to $60 \mathrm{bar}, 17.3 \frac{\mathrm{kg}}{\mathrm{s}}$, and 900 K. Similar to the DLR designs, the ASCR utilizes a 'shell-in-shell' design, with the outer shell containing the system pressure and the inner vessel containing the hot combustion gases [11].

Select university facilities also exist for these complex studies. At the Darmstadt Technical University, an optically accessible combustor was developed for detailed combustion studies at pressures up to 10 bar with a maximum air flow of $0.15 \frac{\mathrm{kg}}{\mathrm{s}}$ heated to $773 \mathrm{~K}$. The experiment is designed as a double walled, air cooled flame tube encased by a pressure vessel [12]. A similar cylindrical quartz section is used to visualize flame dynamics at the high-pressure combustor facility at Pennsylvania State University. Air, preheated up to $870 \mathrm{~K}$, is delivered to a cylindrical combustor at a maximum mass flow rate of $0.27 \frac{\mathrm{kg}}{\mathrm{s}}$ at 24 bar or $0.18 \frac{\mathrm{kg}}{\mathrm{s}}$ at 38 bar $[75,76]$.

\subsection{Facility and Test Stand}

The data presented in this work were collected at the Purdue University Zucrow Laboratories. In the 1940s under the direction of the lab's namesake, Dr. Maurice J. Zucrow, the first laboratories were designed exclusively for rocket propulsion testing. Roughly twenty years later, Professor Arthur Lefebvre overhauled the facilities to accommodate the testing of air-breathing experiments. These changes are most noticeable at the Zucrow High Pressure Lab (HPL) where the current experiment was conducted.

An on site air-plant provides a $0.45 \mathrm{~kg} / \mathrm{s}$ continuous source of clean, dry air compressed to 150 bar. This air-plant feeds into four high pressure air tanks located at 
HPL storing $9000 \mathrm{~kg}$ of air at 150 bar to facilitate higher flow rates. The air is fed into the test cell and split into three independently controlled and metered lines (main, secondary, and tertiary). The main line is can be preheated by a natural-gas fired heat exchanger to provide non-vitiated $40 \mathrm{bar}, 800 \mathrm{~K}$ air at a rate of $4 \mathrm{~kg} / \mathrm{s}$. The secondary line can be directed through an electric heat exchanger capable of heating $0.25 \mathrm{~kg} / \mathrm{s}$ of air at $40 \mathrm{bar}$ to $950 \mathrm{~K}$. The tertiary air line provides an unheated air source for axillary uses, such as a particle seeder. In addition to large compressed air storage, Zucrow Laboratories maintains a significant inert gas supply. Liquid nitrogen from a 2500 gal tank is pumped through a vaporizer at $0.05 \mathrm{~kg} / \mathrm{s}$ to provide a continuous supply of 400 bar gaseous nitrogen. Similar to the air storage, when higher flow rates are required, tankage is available for storing over $9000 \mathrm{~kg}$ of gaseous nitrogen at 40 bar.

Liquid aviation fuels are stored in two 280 gallon flame-shield fuel tanks. From there they are pumped into the test cell at pressures up to 100 bar. Once in the test cell this line splits into two legs for independent control of the main and pilot fuel lines. Each line is equipped with Coriolis flow meters for mass flow measurement and electronically actuated control valves for mass flow control. The test stand is manifolded with low pressure (5 bar) cooling water for flow rates of up to $1.5 \mathrm{~kg} / \mathrm{s}$. High pressure water is supplied at 120 bar to the test stand at $0.7 \mathrm{~kg} / \mathrm{s}$ [7].

\subsection{Experiment Configuration}

The injector used in this experiment was installed in the Purdue high pressure Advanced optical Combustion Experiment (ACE), an optically-accessible, high pressure combustion rig. The design of ACE has been well documented [7,34] and validated with over 150 hours of steady state operation. Previous works have shown temperature and pressure measurement uncertainties to be $0.2 \%$ and $0.04 \%$, respectively. An air mass flow rate uncertainty of $0.48 \%$ and fuel mass flow rate uncertainty of $0.08 \%$. With this is mind, only a brief overview of ACE is given. 


\subsubsection{Test Article}

Figure 3.1 shows a cross section view of the main components in ACE including a breakout of the injector used for this work. Upstream (left) of the shown cross section, preheated high pressure air is provided by the main air system and is metered through an ASME toroidal nozzle. A Laws flow conditioner is installed aft of the sonic nozzle to setup a uniform velocity profile where flow tracers can be introduced to the main flow. A piloted, partially premixed, liquid-fueled swirl burner designed for operation at a high thermal power density is installed against the burner faceplate. The two independently controlled and metered test stand fuel lines are connected to the pilot and main lines on the injector. The pilot line sprays fuel directly into the swirling air flow. The main fuel is premixed with air and swirled outside of the pilot. The water cooled combustion chamber is $230 \mathrm{~mm}$ long and has a $105 \mathrm{~mm}$ square cross-section. Fused quartz windows installed in the walls of the combustion chamber provide a 76 $\mathrm{mm}$ by $100 \mathrm{~mm}$ viewing area beginning at the burner faceplate. The flow area is reduced by half through a water cooled contraction section to close the recirculation zones and provide flame stability. A water cooled mixing section downstream of the contraction, accommodates hardware for exhaust gas sampling. After which, the flow is quenched by the test stand's high pressure water manifold. This is done to reduce the flow temperature to below $600 \mathrm{~K}$ before the flow is back-pressured with an electronically-actuated butterfly valve. A LabView ${ }^{\top M}$ data acquisition system is employed to control the experiment, document conditions, and monitor hardware health. As the experiment is designed for steady-state operation, the flame was run continuously as optical measurements were acquired. The only limiting factor on run duration was fouling of the windows from PIV seed particles. While particle seeding time was minimized to short, ten-second bursts around the actual data acquisition, the total seeding time was limited to approximately 100 seconds at the targeted seeding densities. 


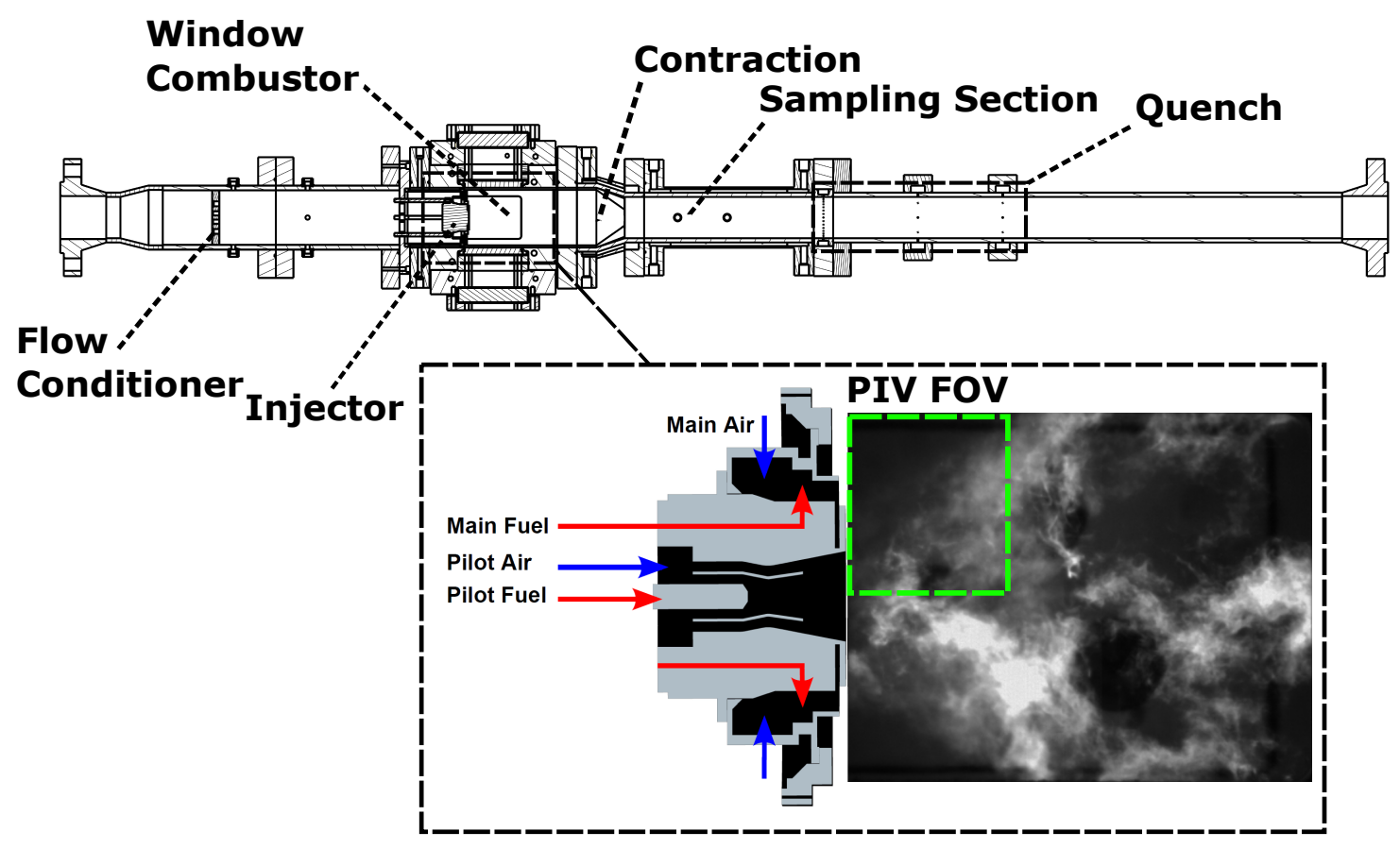

Figure 3.1: Schematic of the combustor with field of view indicated [7].

\subsubsection{Flame}

Based on a survey of run conditions performed previously [7], a single hot fire condition and a base line non-reacting case were chosen for this work. These run conditions are summarized in table 3.1. A Fisher-Tropsch blend (PetroSA PS-150) was chosen as the fuel for the experiment, owing to its extremely low aromatic content $(<60 \mathrm{ppm})$ in comparison with standard Jet-A (18-25\% by volume) which reduces flame luminosity interference for diagnostic purposes. Flame operation was very stable, with no coherent thermoacoustic pulsation. This is partially accredited to the effective damping of the combustion chamber acoustics by the downstream water quench system. 


\begin{tabular}{l|llllll}
$\begin{array}{l}\text { Flame } \\
\text { Condition }\end{array}$ & $\begin{array}{l}\text { Thermal } \\
\text { Power }[\mathrm{kW}]\end{array}$ & $\begin{array}{l}\text { Pressure } \\
{[\mathrm{MPa}]}\end{array}$ & $\begin{array}{l}\text { Main Air Tem- } \\
\text { perature [K] }\end{array}$ & $\phi_{\text {global }}$ & $\phi_{\text {pilot }}$ & $\phi_{\text {main }}$ \\
\hline \hline L1 & 347 & 1.0 & 700 & 0.37 & 1.16 & 0.26 \\
NR & - & 1.0 & 700 & - & - & -
\end{tabular}

Table 3.1: Flame conditions studied

\subsection{Diagnostic Configuration}

As observed in figures 3.2, an angular-displacement SPIV setup was utilized. The cameras were positioned below the center axis and angled upward such that their optical axes intersect at the area of interest in a forward scattering mode. To account for the geometric distortion due to the perspective view, Scheimpflug adapters were used to insure the image, lens, and object planes intersect at a common line [38]. The third camera, used to collect 2-D PIV data, was positioned normal to the flow direction and laser sheet. Temporal synchronization of the lasers and imaging systems was accomplished with a master timing box (Quantum Composers Model 9528). Laser pulse timing was continuously monitored throughout the test using an array of photodiodes and an oscilloscope.

Optical bread-boards are located adjacent the rig for laser manipulation and imaging system mounting. A Linos rail system provides optical access to the top of the experiment on stable platform. Two horizontal rails extend over the rig to facilitate multiple simultaneous diagnostics. A two-axis translational system provides the capability to traverse the measurement probe volume within the flow, facilitating flow-field mappings and detailed diagnostics. The detection systems are also mounted on two-axis translational system, with dependent control on the probe volume traverse to maintain system focus and signal strength. All optical and optomechanical systems are operated remotely from the experiment control room during testing; as is consistent with test rig operating procedures. 


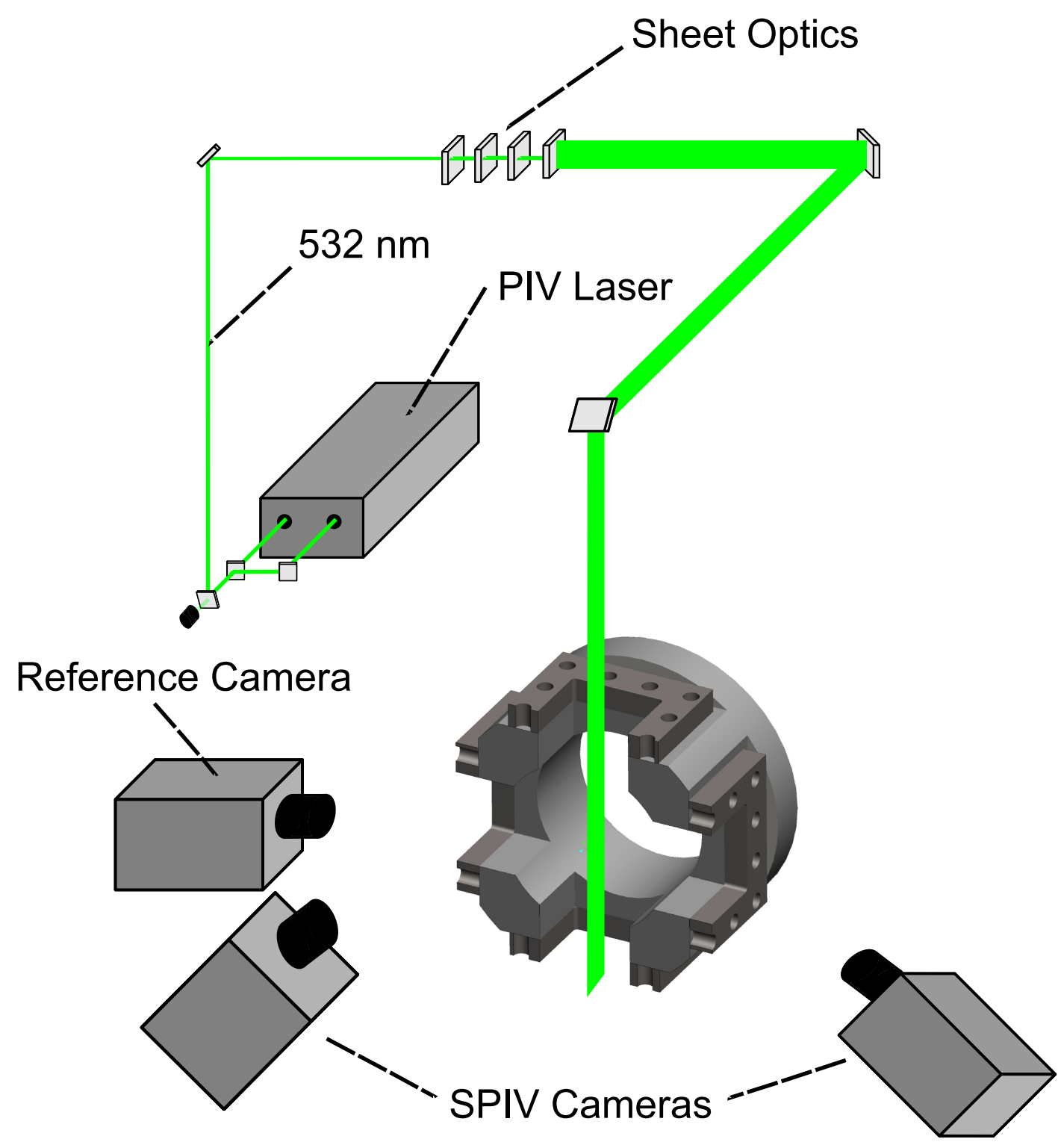

Figure 3.2: Schematic diagram of diagnostics configuration.

\subsubsection{Illumination and Optics}

A dual-cavity, diode-pumped, solid state Nd:YAG laser (Edgewave IS811-DE) provided $532 \mathrm{~nm}$ light at $6 \mathrm{~mJ} /$ pulse with a repetition rate of $6 \mathrm{kHz}$ for the SPIV and PIV measurements. The beam was expanded to approximately $30 \mathrm{~mm}$ in height using two cylindrical lenses $\left(f_{P I V, 1}=-25 \mathrm{~mm}, f_{P I V, 2}=450 \mathrm{~mm}\right)$ in a cylindrical telescope 
arrangement. Two positive cylindrical lenses $\left(f_{P I V, 3: 4}=150 \mathrm{~mm}\right)$ were used to focus the collimated sheet just past the test section to reduce particle dropout near the beam waist. The average sheet thickness in the test section was measured to be 650 $\mu \mathrm{m}$ full-width half-max (FWHM). The inter-pulse separation time was set to $4 \mu \mathrm{s}$.

\subsubsection{Particle Seeder}

A fluidized bed was used to seed $\mathrm{TiO}_{2}$ particles (250 nm nominal mean diameter) into the main air flow. The particles were introduced approximately four hydraulic diameters upstream of the burner using an injector that spanned the width of the inlet section.

\subsubsection{Stereoscopic Particle Image Velocimetry}

The Mie scattering signal from the particle field was collected through two $200 \mathrm{~mm}$ focal length, f/4 objective lenses (AF Micro-Nikkor IF-ED) and recorded with two high-speed CMOS cameras (Photron SA-4) with a resolution of $496 \times 557$ pixels. This resulted in an image resolution of $58.6 \mu \mathrm{m} /$ pixel in the raw scattering images. The cameras had sufficient on-board memory to acquire 3000 double-frame image pairs per run. A custom ultra-steep bandpass filter (3 nm FWHM centered on $532 \mathrm{~nm}$ ) was used to maximize the SNR in the highly luminous flame environments. Image calibrations, scaling, and particle stereo cross-correlations were performed using the multi-pass adaptive window offset cross-correlation algorithm in the LaVision commercial software (DaVis 8.2.3). Spatial calibration data was collected by imaging a dual-sided, dual-plane dot target (LaVision Type 05).

Results presented in this work were processed using a final window size of $24 \mathrm{x}$ 24 pixels with $50 \%$ overlap. The corresponding spatial resolution is $1.406 \mathrm{~mm}$ with vector spacing of $0.703 \mathrm{~mm}$. A local median filter, based on a 3x3 square kernel, was used to remove spurious vectors beyond a specified threshold and replaced with an 
interpolated vector. The number of spurious vectors filtered from the instantaneous images was consistently less then five percent of the total vector count.

\subsubsection{2-D Particle Image Velocimetry}

A third high-speed CMOS camera (Phantom v411), positioned normal to the laser sheet, recorded the Mie scattering from the $\mathrm{TiO}_{2}$ particles through a $105 \mathrm{~mm}$ focal length, f/2.8 objective lens (Nikkor AF-S Micro) with a resolution of 481 x 585 pixels. The camera had sufficient on-board memory to acquire 3000 double-frame image pairs per run. Again, a custom ultra-steep bandpass filter (3 nm FWHM centered on 532 $\mathrm{nm})$ was used to maximize the SNR. The camera was positioned to reflect the same field of view as that of the SPIV cameras shown in figure 3.1, this resulted in an image resolution of $61.6 \mu \mathrm{m} /$ pixel in the raw scattering images. Image calibrations, scaling, and particle cross-correlations were performed using the multi-pass adaptive window offset cross-correlation algorithm in the LaVision commercial software (DaVis 8.2.3). Spatial calibration data was collected by imaging a dual-sided, dual-plane dot target (LaVision Type 05).

Results presented in this work were processed using a final window size of $24 \mathrm{x}$ 24 pixels with $50 \%$ overlap. The corresponding spatial resolution is $1.478 \mathrm{~mm}$ with vector spacing of $0.739 \mathrm{~mm}$. A local median filter, based on a 3x3 square kernel, was used to remove spurious vectors beyond a specified threshold and replaced with an interpolated vector. The number of spurious vectors filtered from the instantaneous images was consistently less then five percent of the total vector count. 


\section{RESULTS AND DISCUSSION}

As described previously, in high-power density systems, measurements can be very challenging. Two examples of this can be found in the data presented here. The first is that found in figure 4.1. The image on the left is a single raw image of the seeded particle field acquired during the reacting test case. Located at roughly 15 $\mathrm{mm}$ in the positive -y direction and extending from the $10 \mathrm{~mm}$ to $20 \mathrm{~mm}$ in the axial direction is a block of corrupt or damaged pixels. Even when a background image was acquired with the lens cap installed, the block was present. During image preprocessing, various filters and masks were applied in an attempt to mitigate the effects of the block on the vector generation. Applying a geometric mask proved ineffective, as the corrupt area is too large to accurately interpolate over. Instead, the cross correlation algorithim within DaVis treated the mask as an obstruction within the flow generating erroneous vectors. A background subtraction filter yielded a similar result. Without the ability to correct for this error, the resulting velocity field has a clear discontinuity as can be seen in the right image of figure 4.1.

The second issue involves a reflection only captured by one of the stereo cameras. Figure 4.2 shows a raw image from this camera during a reacting case (The reflection is present in the non-reacting case as well but is not nearly as prominent). Reflections are common and sometimes unavoidable when performing diagnostics in confined, windowed test articles and usually can be corrected for during image pre-processing. However, the presence of the reflection in only one camera caused issues during the reconstruction of the third velocity component. As in previous efforts both static masks and background filters only exacerbated the problem. The vector fields shown in figure 4.2 were generated without any attempts to correct for the reflection.

The remainder of the vector fields (both 2-component and 3-component) presented in this chapter were generated with out explicit image pre-processing to mitigate these 


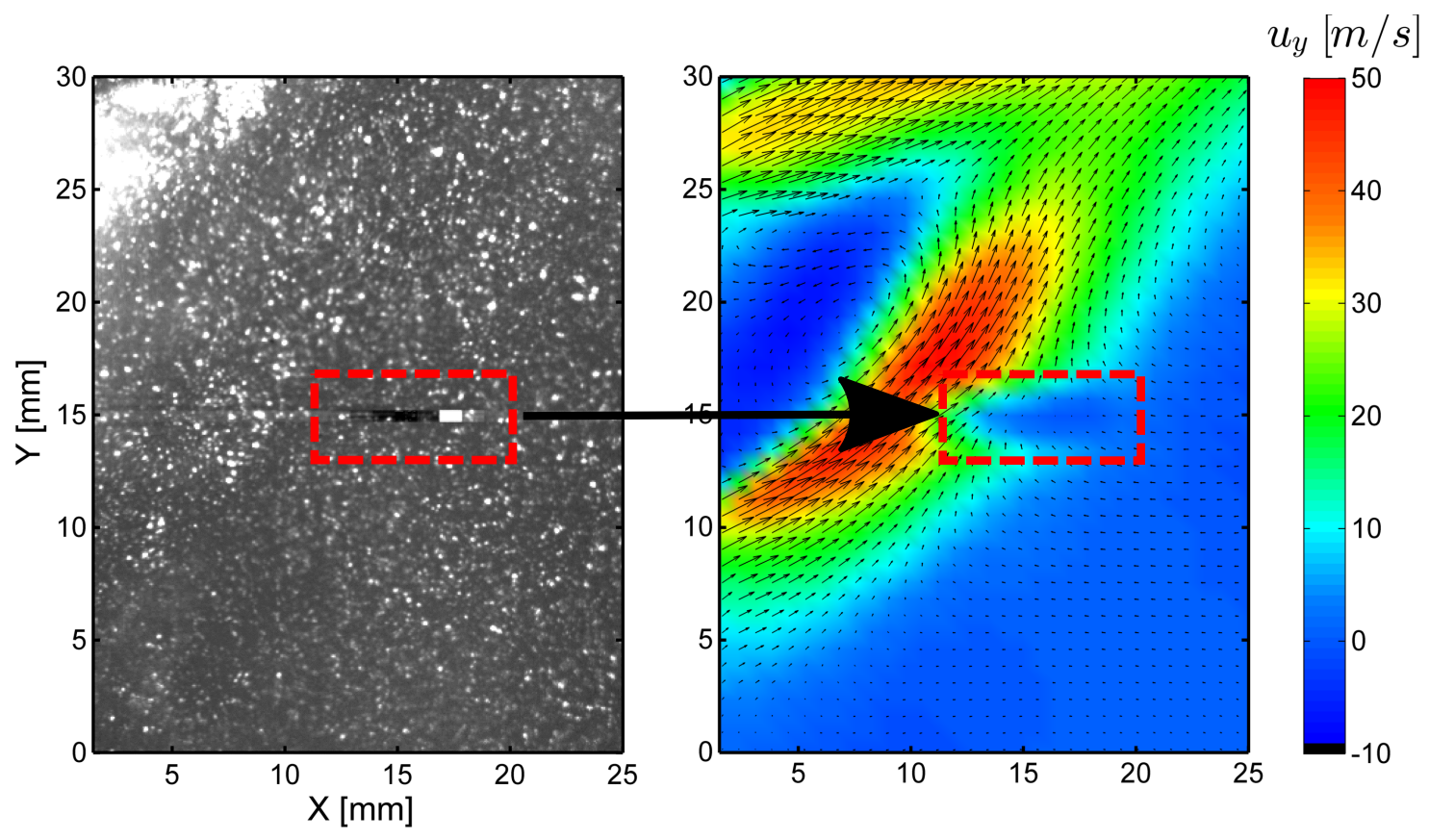

Figure 4.1: Raw image (left) of particle field (reacting case) showing location of damaged pixels and it's effect on the average vector field (right)

errors. These regions were specifically avoided when comparing the SPIV measurements to that of the PIV. 


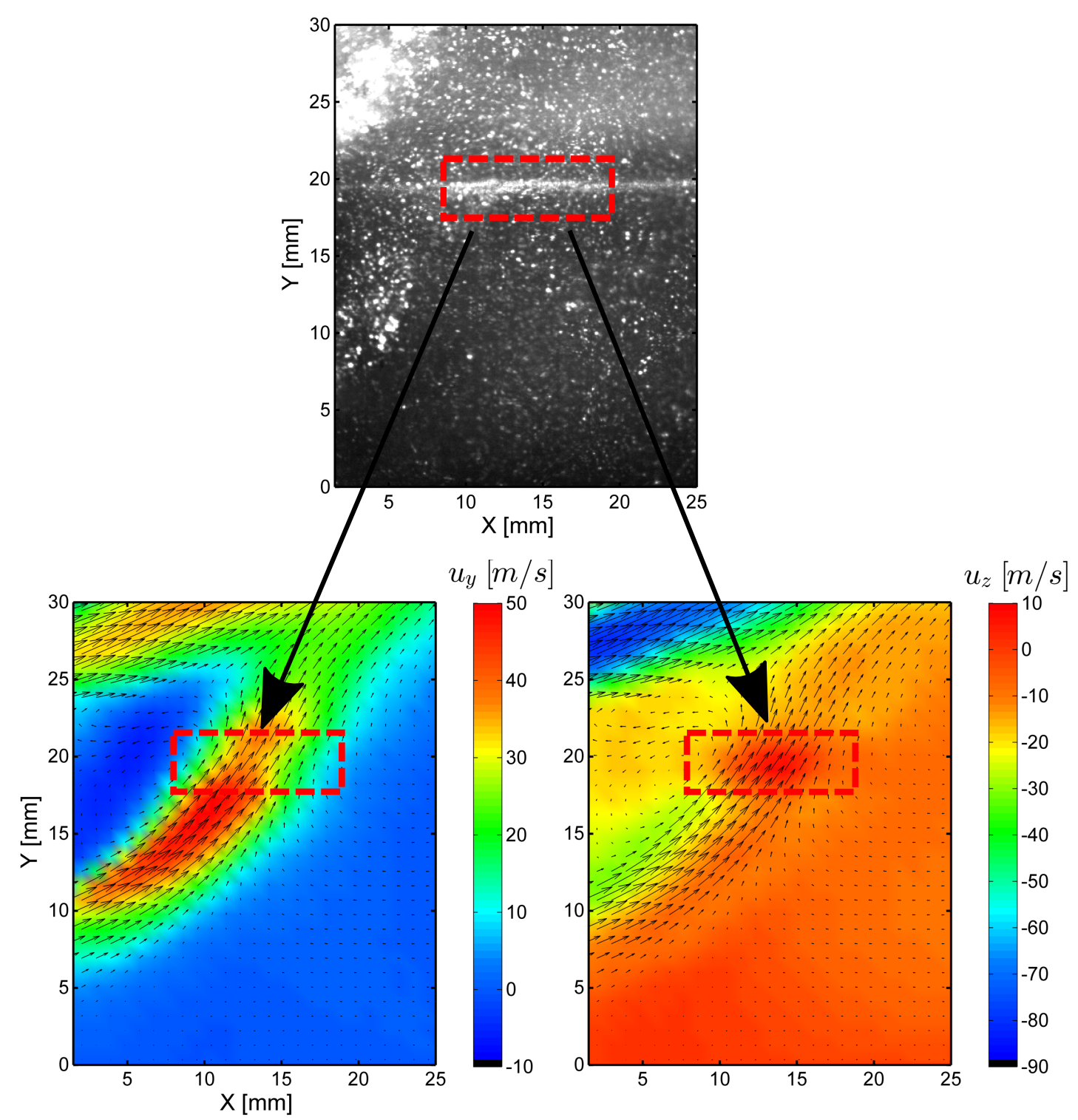

Figure 4.2: Raw image (top) of particle field (reacting case) showing location of reflection and its effect on the average $U_{y}$ and $U_{z}$ vector fields (bottom)

\subsection{Mean Comparison}

As a baseline measure of comparison, the time average of the axial $\left(U_{x}\right)$ and radial $\left(U_{y}\right)$ velocities measured by the SPIV system were spatially subtracted from the corresponding PIV velocity measurements. The resulting magnitude difference is presented in figure 4.3 for the non-reacting condition and figure 4.4 for the reacting 

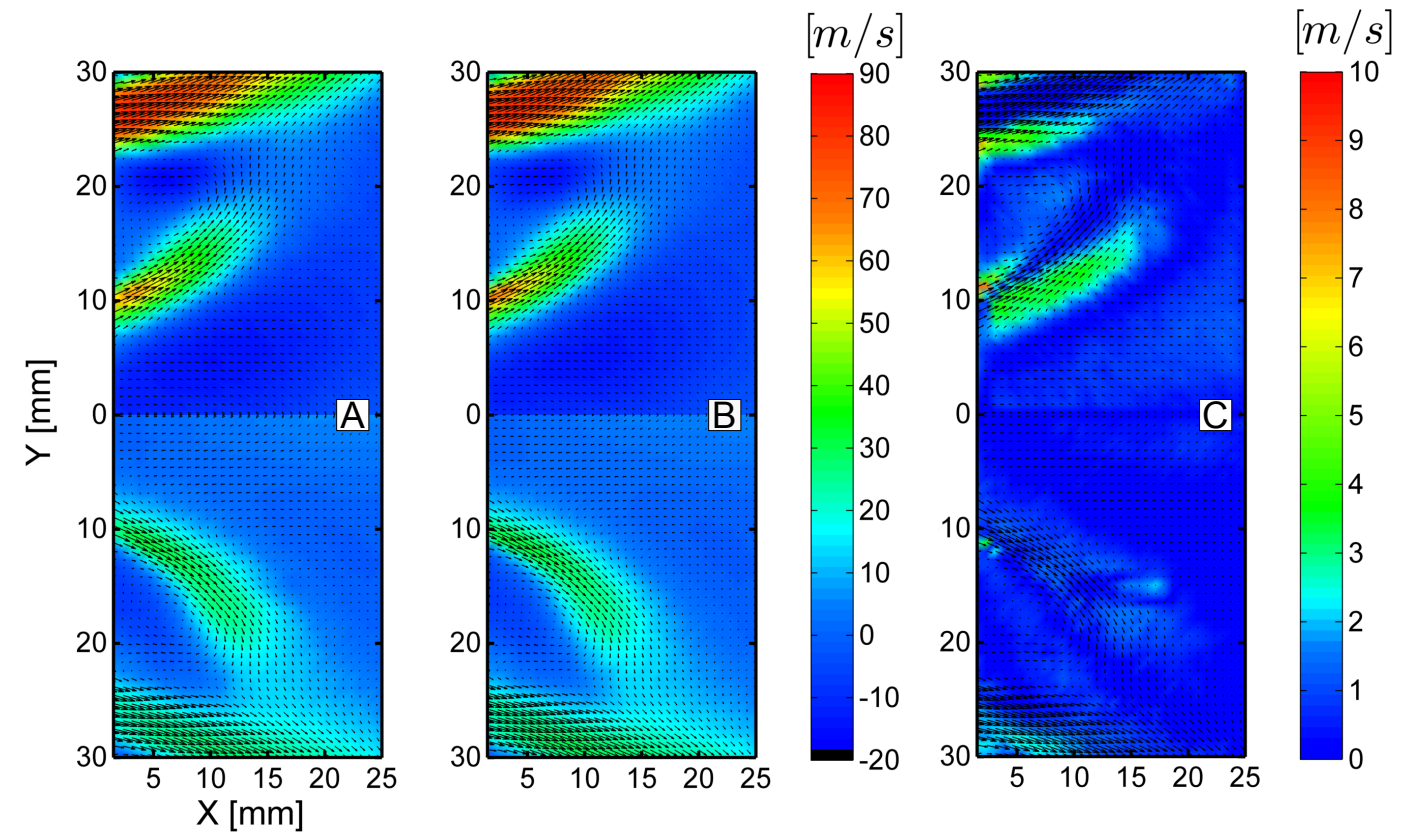

Figure 4.3: Non-reacting case mirrored mean velocity fields $\left(U_{x}\right.$ on top and $U_{y}$ on bottom): (A) PIV (B) SPIV (C) magnitude of the difference between (A) and (B)

case. Each image is mirrored across the center line, with the contours on the top half representing the axial velocity magnitudes and the bottom half representing the radial velocity magnitudes.

Referencing the burner design in figure 3.1, the region of high axial velocity at $y$ $=27 \mathrm{~mm}$ in figure 4.3 is the main jet. At approximately $\mathrm{y}=10 \mathrm{~mm}$ the pilot jet is introduced. These two swirling jets converge roughly $15 \mathrm{~mm}$ downstream of the burner resulting in a recirculation zone between the two. Near the center line a low negative axial velocity is observed, evidence of a potential inner-recirculation zone. The difference in velocities shown in figure $4.3(\mathrm{C})$ indicate that the two measurement techniques disagree the most in the areas of high velocity gradients. It is hypothesized this is due to the highly three dimensional nature of the shear layer between the coaxial swirling flows, causing a bias error in the PIV measurement as a result of the projection of the out of plane component onto the image plane. 

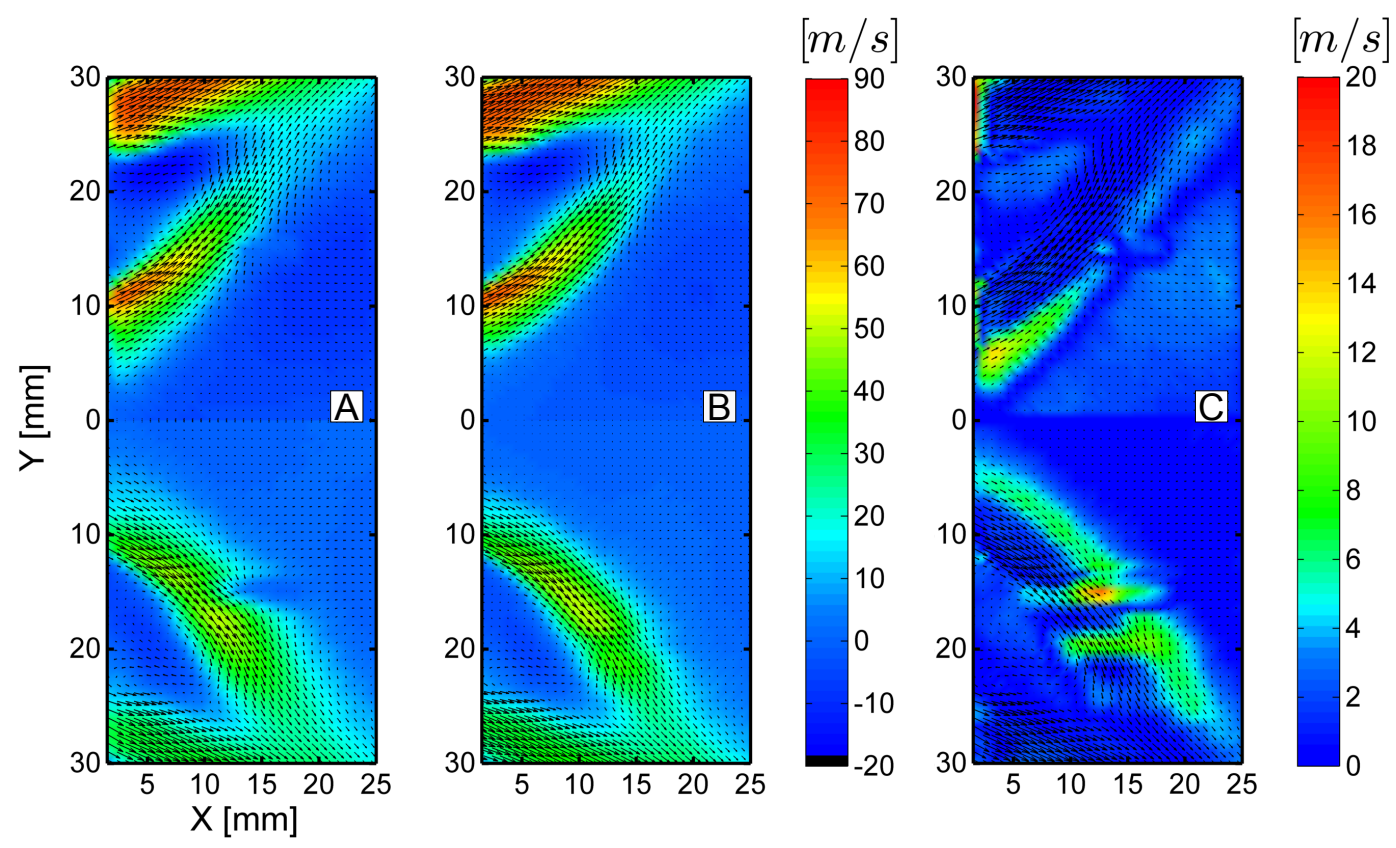

Figure 4.4: Reacting case mirrored mean velocity fields ( $U_{x}$ on top and $U_{y}$ on bottom): (A) PIV (B) SPIV (C) magnitude of the difference between (A) and (B)

The mean comparison in the reacting case shown in figure 4.4 presents a similar result. The velocity measurements of the shear layer provide the most significant discrepancies. As was mentioned earlier, the reflection and corrupt pixel errors significantly influence the accuracy of the vector generation and are much more noticeable in the reacting case data. This is attributed to window fouling caused by seeding particles agglomerating to the thermally softened windows, thus reducing the signalto-noise ratio.

\subsection{Time Series comparison}

Beyond the mean structure comparison it is useful to compare the vector fields generated by the individual diagnostics on a shot-to-shot basis. Figures 4.5 and 4.6 show five sequential vector fields for the non-reacting and reacting case, respectively. Based on the hypothesis generated when reviewing the mean structure, the regions of 

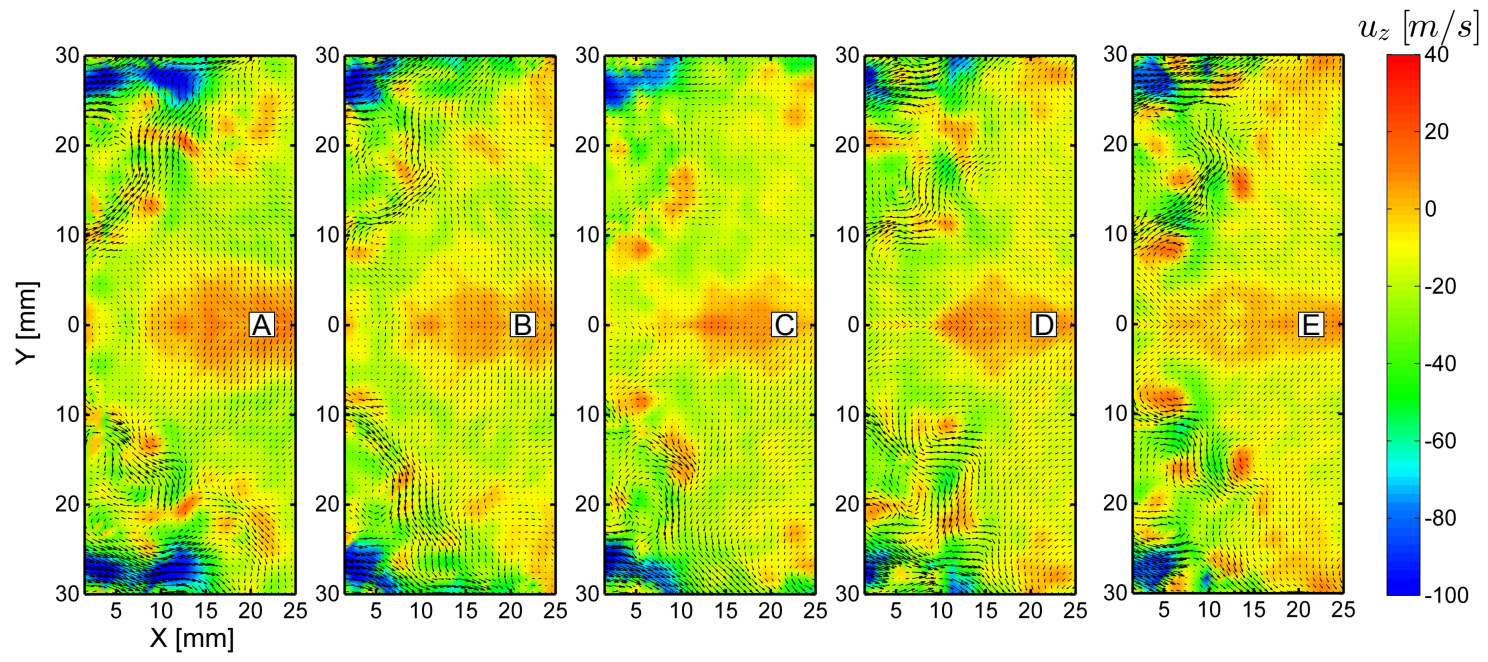

Figure 4.5: Non-reacting case time series of mirrored velocity fields (PIV above the center line and SPIV below) overlaid on a contour plot of the azimuthal velocity $U_{z}$

the flow with the greatest azimuthal velocity should reflect the greatest difference in vectors generated between the two diagnostics. This is clearly observed in the main jet region of figure 4.6(D).

As noted earlier, the discrepancy between the SPIV and PIV velocity magnitudes was greater in the mean reacting case, than the non-reacting. Looking at the plot of the azimuthal velocity magnitude shows that the reacting case has much more out of plane motion near the pilot jet than the non-reacting case this supports the hypothesis of the bias error.

In order to validate the observations made in both the mean and time series comparisons, a more detailed analysis is required. 

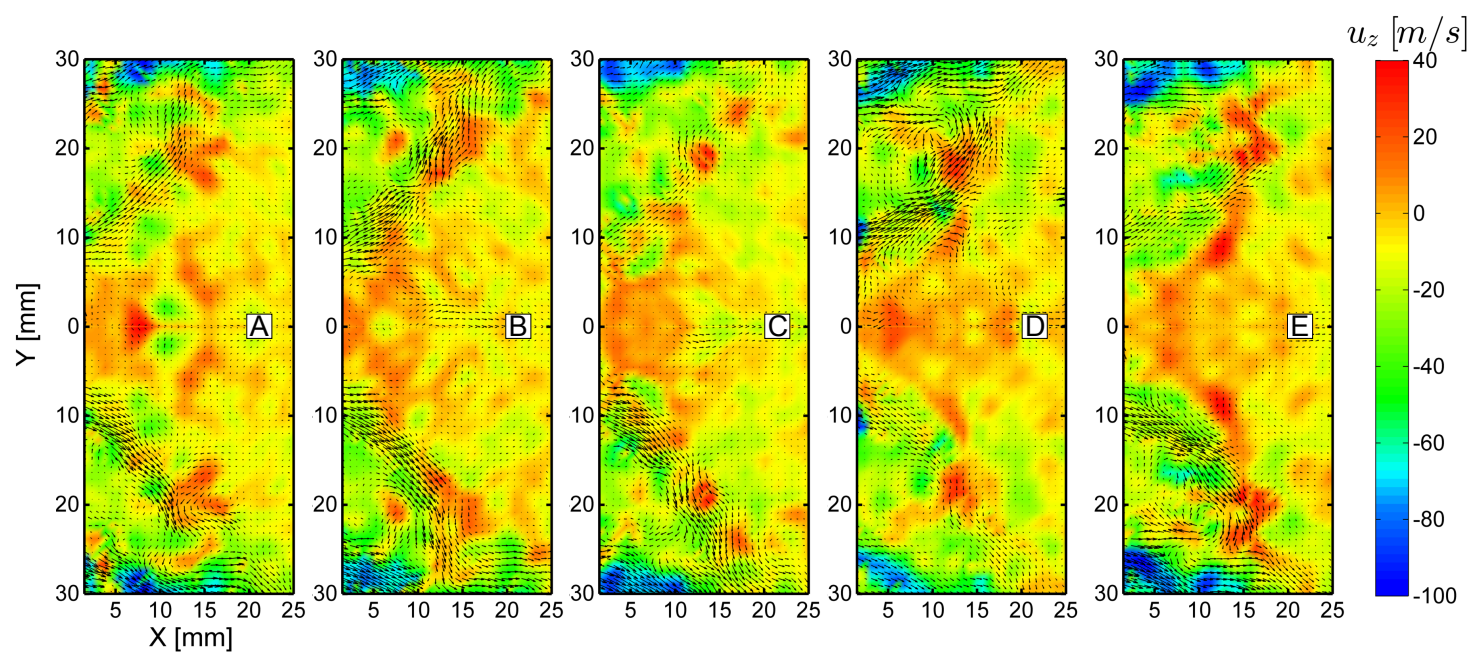

Figure 4.6: Reacting case time series of mirrored velocity fields (PIV above the center line and SPIV below) overlaid on a contour plot of the azimuthal velocity $U_{z}$

\subsection{Point Comparison}

Four specific locations in the flow field were chosen to perform more in depth comparisons of the results from the two measurement techniques. These probe locations are shown in figures 4.7-4.12. Probe A (a) is located in the main jet, B (b) is in the jet recirculation zone, $\mathrm{C}(\mathrm{c})$ is in the pilot jet, and $\mathrm{D}(\mathrm{d})$ is positioned in the inner recirculation zone.

\subsubsection{Probability density function}

From the mean comparisons presented earlier, on average, the two diagnostics appear to be in good agreement. This can be misleading. A clearer metric is to compare the temporal velocity distributions at defined locations for each diagnostic. This is best accomplished through a comparison of probability density functions (PDF) of the measurements acquired at each probe location. In figure 4.7 the PDF's for the axial velocities in the non reacting case show strong agreement. For example figures 4.7(A) and (a) both have a negative skew distribution, whereas points at location (B) of the same figure follow a normal distribution. The same can be said for all the 

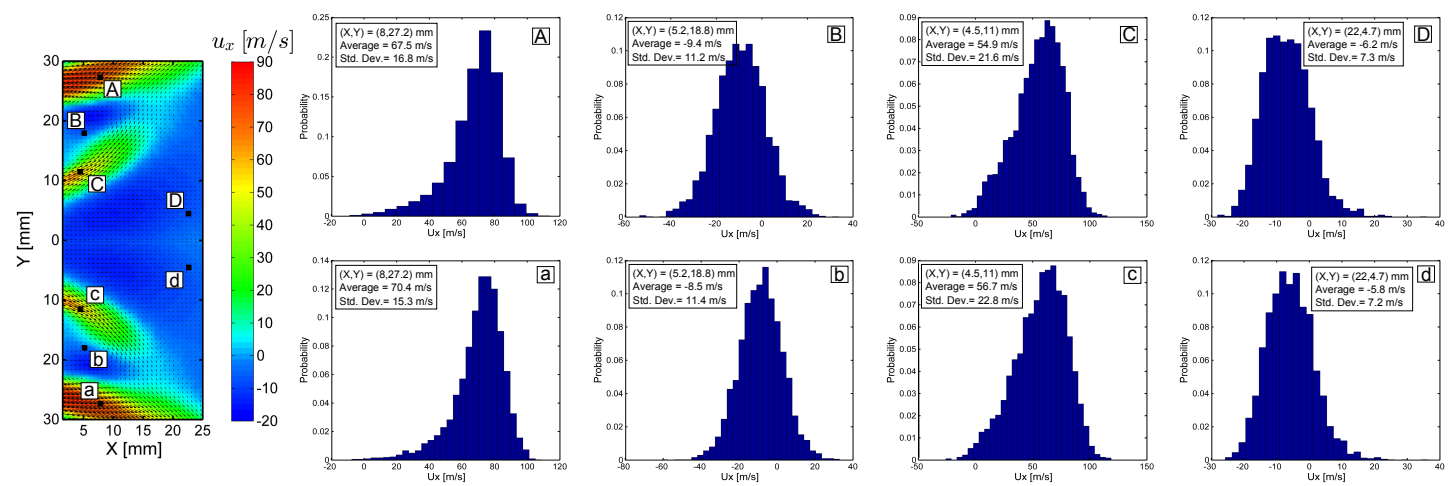

Figure 4.7: PDF for $U_{x}$ in the non-reacting case: (A-D) PIV results (a-d) SPIV results
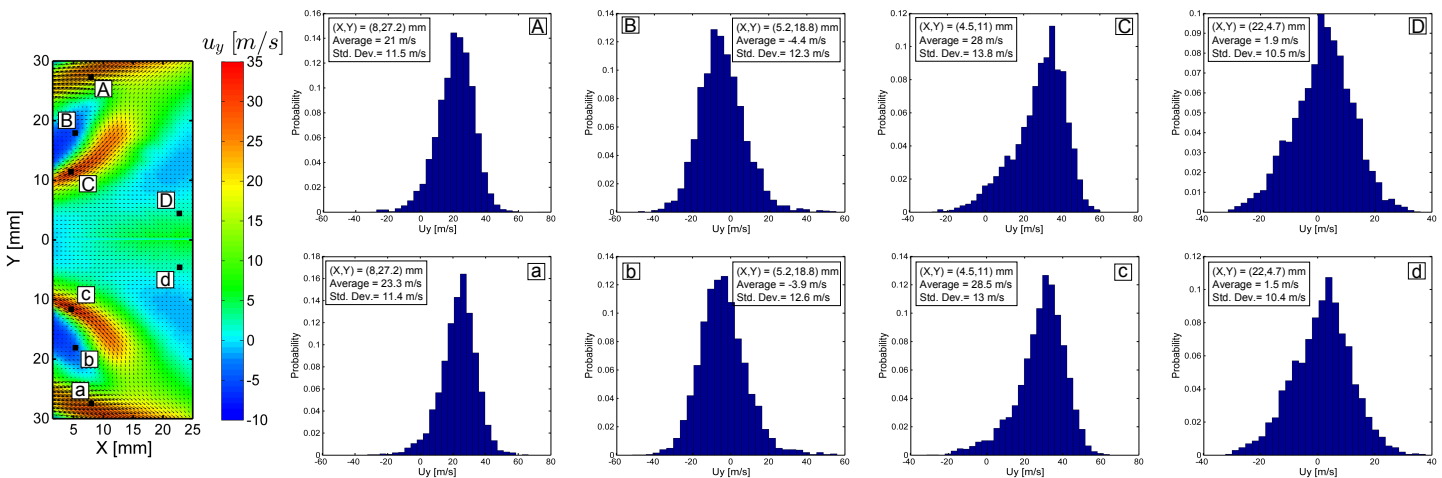

Figure 4.8: PDF for $U_{y}$ in the non-reacting case: (A-D) PIV results (a-d) SPIV results

non-reacting PDFs. Regardless of the measurement technique the vector choice at these locations have similar probability distributions.

This trend continues when taken to the reacting case. The main jet axial velocity PDF again shows a negative skew distribution. When looking more closely at the locations of the probes in the mean velocity field, the explanation for the shape of the distributions becomes more evident. Probe A is located near the shear layer of the main jet and as was seen in the time series figures the core structure of high axial velocities fluctuates spatially. Since the probe is located near the edge of this jet, it follows that the probe would have a bias towards finding velocities outside the jet. 

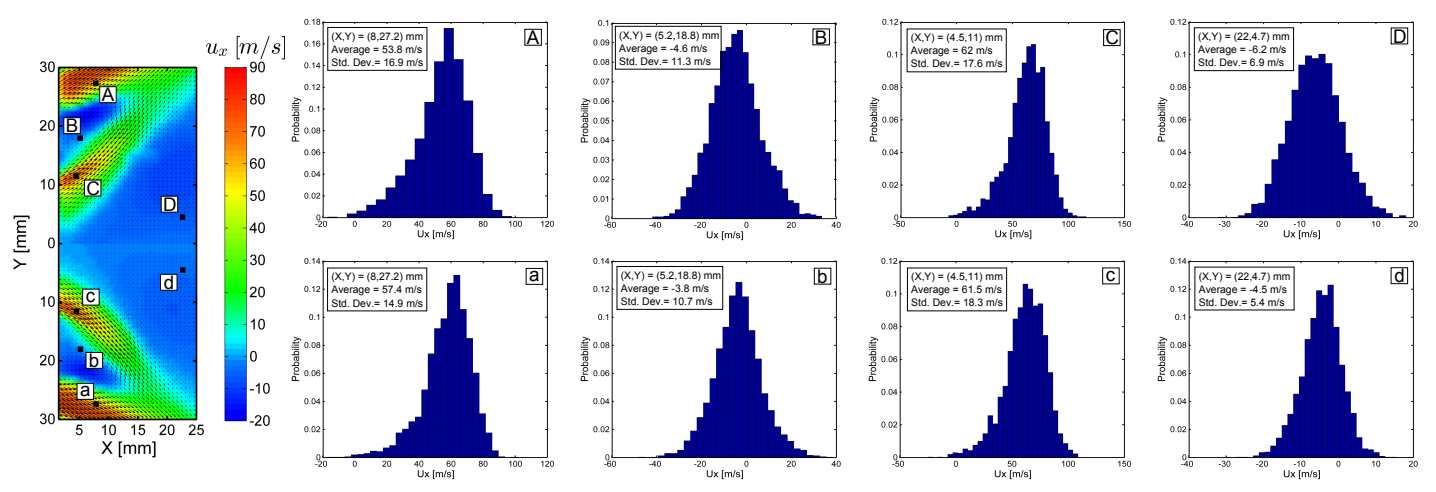

Figure 4.9: PDF for $U_{x}$ in the Reacting case: (A-D) PIV results (a-d) SPIV results
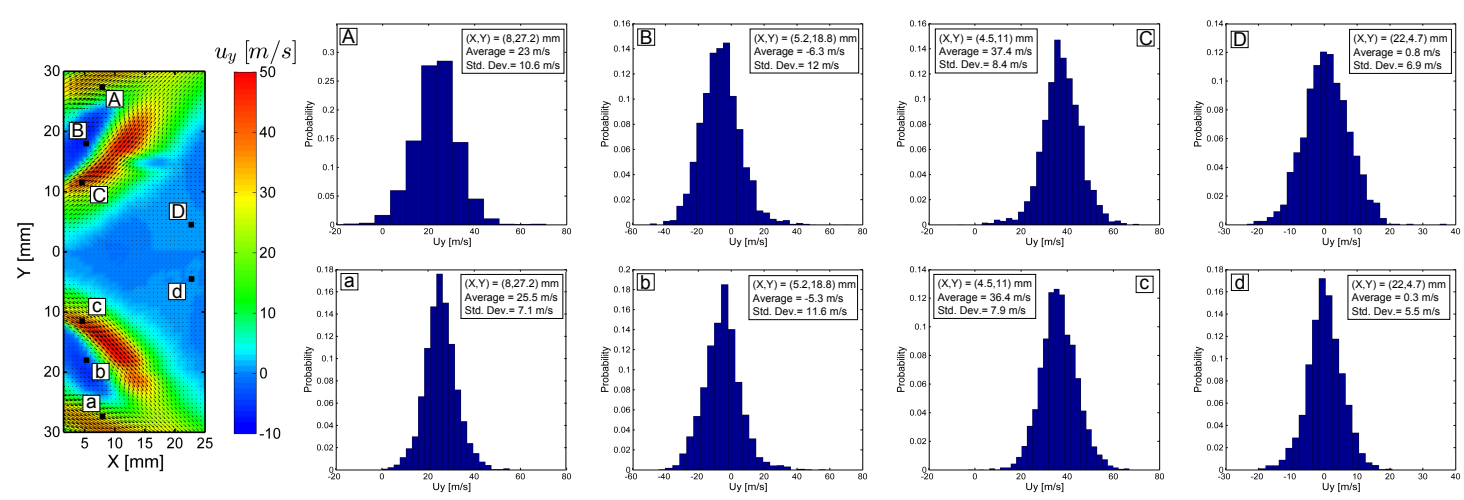

Figure 4.10: PDF for $U_{y}$ in the Reacting case: (A-D) PIV results (a-d) SPIV results

The same logic can be applied to probe locations B and D. The mean images suggest that the probes are not located in regions of any significant velocity gradients. As such the their probability distributions are normal.

\subsubsection{Correlation strength and peak ratio}

Taking a step deeper into the validity of the data, the correlation strengths and peak ratios of the probe locations are compared at a single point in time. The correlation strength as output by $\mathrm{DaVis}$ ranges from 0 - 1.0. It is the normalized value of the correlation peak height within each window. Generally the closer the correlation value is to 1 , the higher the confidence in the vector. This can be misleading though, in 
flows with significant background, like that found in this study, the correlation values are all relatively low (less than 0.6). If all vectors with low correlations strength were deemed invalid the majority of the flow field would be rejected. A better indicator of the validity of a displacement vector is the peak ratio, Q. As mentioned in chapter two the peak ratio is the height of the highest peak in the correlation plane divided by the second highest peak in the correlation plane, this is an indirect measure of the correlation SNR. For good PIV data a Q-factor between 3-4 (or greater) is expected. DaVis provides the peak ratio relative to the correlation plane background, this is shown in equation

$$
Q=\frac{P 1-\min }{P 2-\min }
$$

where P1 and P2 are the highest and second highest peak heights respectively and $\min$ is the lowest value of the correlation plane. This relative peak ratio helps to compensate for the background noise. When DaVis can not determine a distinct second peak, it returns a value of 100. Since the peak search is done within DaVis, it is unclear how it discerns the presences of a second peak. As is a common theme when working with commercial software, there is an inherent level of uncertainty in the validity of the calculations or assumptions made within the software as these methods are not made clear (the "black box" issue).

With this foreknowledge, correlation maps were generated in DaVis for each probe location. For each test case, the correlation maps for both diagnostics were collected at the same point in time and space. Additionally the correlation values and peak ratios were extracted for each probe location and are reported in tables 4.1 and 4.2.

Comparing the correlation maps with the peak ratios output by DaVis reinforces the short comings of performing vector generation algorithms with a commercial software. For example, Location B in the non reacting case has a peak ratio of 100 . As stated earlier, this means DaVis did not find a second peak within the interrogation 


\begin{tabular}{l|llll} 
Probe Location & $\begin{array}{l}\text { Correlation } \\
\text { Value (PIV) }\end{array}$ & $\begin{array}{l}\text { Peak } \\
\text { Ratio } \\
(\text { PIV) }\end{array}$ & $\begin{array}{l}\text { Correlation } \\
\text { Value (SPIV) }\end{array}$ & $\begin{array}{l}\text { Peak } \\
\text { Ratio } \\
(\text { SPIV) }\end{array}$ \\
\hline \hline A(a) & 0.282 & 100 & 0 & 1.0 \\
B(b) & 0.291 & 100 & 0.295 & 50.8 \\
C(c) & 0.346 & 1.675 & 0.276 & 100 \\
D (d) & 0.588 & 100 & 0.62 & 100
\end{tabular}

Table 4.1: Correlation values and peak ratios generated at each probe location for the non-reacting case

\begin{tabular}{l|llll} 
Probe Location & $\begin{array}{l}\text { Correlation } \\
\text { Value (PIV) }\end{array}$ & $\begin{array}{l}\text { Peak } \\
\text { Ratio } \\
(\text { PIV) }\end{array}$ & $\begin{array}{l}\text { Correlation } \\
\text { Value (SPIV) }\end{array}$ & $\begin{array}{l}\text { Peak } \\
\text { Ratio } \\
\text { (SPIV) }\end{array}$ \\
\hline \hline A(a) & 0.216 & 1.2 & 0.281 & 1.43 \\
B(b) & 0.466 & 100 & 0.465 & 100 \\
C (c) & 0.414 & 100 & 0.318 & 50.84 \\
D(d) & 0.458 & 100 & 0.461 & 2.564
\end{tabular}

Table 4.2: Correlation values and peak ratios generated at each probe location for the reacting case

window. This seems counterintuitive when looking at the correlation map for location (B) in figure 4.11. The map appears to have two clear peaks. However, this could be a result of poor resolution with respect to the flow scales present in the window, these two peaks could actually be part of a single broader peak. Conversely locations (D) and (d) of the non reacting case, have clearly defined single peaks with high correlation values with a corresponding peak ratio. These high correlation values are indicative of the type of flow the probe is located in. The flow around probe (D) is very uniform in magnitude and direction. Where as the flow at locations $(\mathrm{C})$ and $(\mathrm{c})$ appears to have a large velocity gradient. This could be the reason for what appears to be two peaks in the correlation maps. It is also interesting to note that the axial velocities of the flow surrounding probes (A) and (a) are quite different despite being recorded at the same time and position. As has been noted earlier this is likely due 

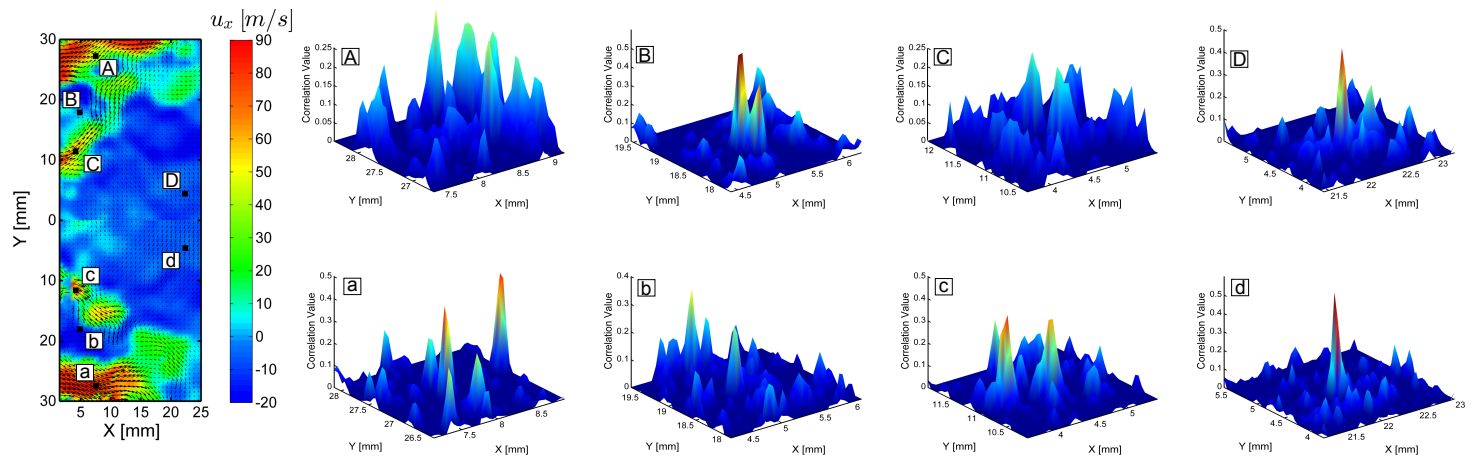

Figure 4.11: Correlation maps generated at each probe location for the non-reacting case
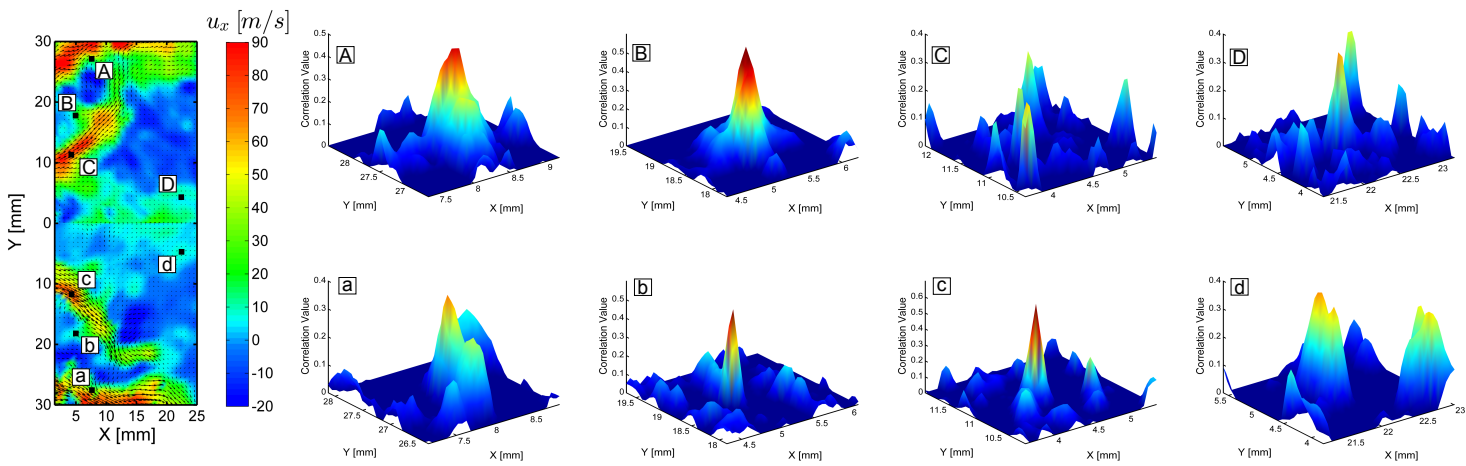

Figure 4.12: Correlation maps generated at each probe location for the reacting case

to the implicit out of plane bias error, of 2-component PIV measurements taken in three dimensional velocity flows.

For the reacting case the information extracted from the probe locations is equally insightful. The main peak in the correlation maps of (A) and (a) seem to have a similar broadening characteristics which can be found when the particle displacements within an interrogation window have a significant difference in magnitude. These quick changes in displacement over a small distance are evidence of a steep velocity gradient or shear layer. Despite the unknown in regards to the peak search in DaVis, the correlation maps provide a good method of validating observations in the vector fields. 


\section{CONCLUSIONS}

Particle Image Velocimetry is a powerful non-intrusive diagnostic that can provide temporally and spatially resolved velocity field measurements in a variety of fluid mediums. When applied to reacting flows, consideration must be given to the effects of the heat release on the measurements capabilities. The advantages of Stereoscopic Particle Velocimetry over traditional two-component PIV were introduced and the methods by which both of these diagnostics are accomplished were discussed. The configuration of the Purdue Aviation Combustion Experiment at Zucrow Laboratories has been described. The Diagnostic systems were presented as established for this work. The test matrix and the field of view over which the diagnostics were performed was given.

Stereoscopic particle image velocimetry measurements were successfully collected at a $6 \mathrm{kHz}$ in a liquid fueled combustor operating at realistic engine conditions. The SPIV results were compared with 2-component PIV results that were collected simultaneously. General agreement in the mean structures was found. Discrepancies in the measurements were hypothesized to be a function of the out of plane biasing inherent with 2-component PIV. The shortcomings of using a commercial "black box" software for vector generation were exposed. The inability to validate the individual steps taken by the software to generate the vectors creates an inherent uncertainty in the measurements. Still the ability of the SPIV technique to resolve the third out of plane component was shown to be a more robust measurement when applied to highly three dimensional flows. 
LIST OF REFERENCES 


\section{REFERENCES}

[1] Hukam C. Mongia. N+3 and N+4 Generation Aeropropulsion Engine Combustors: Part 4: Specific Fuel Burn and $\mathrm{CO}_{2}$ Emission. In AIAA Joint Propulsion Conference, AIAA-2013-3652, San Jose, CA, July 14-17, 2013.

[2] Chi-Ming Lee and Clarence Chang. NASA Project Develops Next Generation Low-Emissions Combustor Technologies. In AIAA Aerospace Science Meeting, AIAA-2013-0540, Grapevine, TX, January 007-10, 2013.

[3] ICAO. Report of the independent experts on the LTTG NOx review and medium and long term technology goals for NOx. ICAO Document 9887, 2008.

[4] C.E. Johnson and R.G. Derwent. Relative radiative forcing consequences of global emissions of hydrocarbons, carbon monoxide and NOx from human activities estimated with a zonally-averaged two-dimensional model. Climatic Change, 34(3-4):439-462, 1996.

[5] S.M. Correa. A Review of NOx Formation Under Gas-Turbine Combustion Conditions. Combustion Science and Technology, 87(1-6):329-362, 1993.

[6] S.R. Turns. An Introduction to Combustion: Concepts and Applications. McGraw-Hill Higher Education, 2000.

[7] Carson D. Slabaugh, Andrew C. Pratt, Robert P. Lucht, Scott E. Meyer, Michael Benjamin, Kent Lyle, and Mark Kelsey. The development of an opticallyaccessible, high-power combustion test rig. American Institute of Physics: Review of Scientific Instruments, 85(3), 2014. doi: 10.1063/1.4867084.

[8] David J. Beerer and Vincent G. McDonell. An experimental and kinetic study of alkane autoignition at high pressures and intermediate temperatures. Proceedings of the Combustion Institute, 33(1):301-307, 2011.

[9] J.C. Broda, S. Seo, R.J. Santoro, G. Shirhattikar, and V. Yang. An experimental study of combustion dynamics of a premixed swirl injector. Symposium (International) on Combustion, 27(2):1849-1856, 1998.

[10] P Griebel, P Siewert, and P Jansohn. Flame characteristics of turbulent lean premixed methane/air flames at high pressure: Turbulent flame speed and flame brush thickness. Proceedings of the Combustion Institute, 31(2):3083-3090, 2007.

[11] Yolanda R Hicks, Randy J Locke, and Robert C Anderson. Optical measurement and visualization in high-pressure high-temperature aviation gas turbine combustors. Symposium on Applied Photonics, pages 66-77, 2000.

[12] B Janus, A Dreizler, and J Janicka. Experimental study on stabilization of lifted swirl flames in a model GT combustor. Flow, Turbulence and Combustion, 75(1-4):293-315, 2005. 
[13] A. Lantz, R. Collin, J. Sjöholm, Z. S. Li, P. Petersson, and M. Alden. High-Speed Fuel/Hydroxyl Radical Imaging in a Gas Turbine Pilot Burner. AIAA Journal, 50(4):971-976, 2012.

[14] TM Muruganandam, B-H Kim, MR Morrell, V Nori, M Patel, BW Romig, and JM Seitzman. Optical equivalence ratio sensors for gas turbine combustors. Proceedings of the Combustion Institute, 30(1):1601-1609, 2005.

[15] Ulrich Stopper, Manfred Aigner, Wolfgang Meier, Rajesh Sadanandan, Michael Stohr, and Ik Soo Kim. Flow Field and Combustion Characterization of Premixed Gas Turbine Flames by Planar Laser Techniques. Journal of Engineering for Gas Turbines and Power, 131(2), 2009.

[16] Sulabh K. Dhanuka, Jacob E. Temme, James F. Driscoll, and Hukam C. Mongia. Vortex-shedding and mixing layer effects on periodic flashback in a lean premixed prevaporized gas turbine combustor. Proceedings of the Combustion Institute, 32(2):2901-2908, 2009.

[17] Isaac Boxx, Michael Stohr, Campbell Carter, and Wolfgang Meier. Sustained multi-kHz flamefront and 3-component velocity-field measurements for the study of turbulent flames. Applied Physics B: Laser and Optics, Rapid Communication, 95:23-29, 2009.

[18] Isaac Boxx, Christoph M. Arndt, Campbell D. Carter, and Wolfgang Meier. High-speed laser diagnostics for the study of flame dynamics in a lean premixed gas turbine model combustor. Experiments in Fluids, 52(3):555-567, 2010.

[19] Isaac Boxx, Michael Stohr, Campbell Carter, and Wolfgang Meier. Temporally resolved planar measurements of transient phenomena in a partially pre-mixed swirl flame in a gas turbine model combustor. Combustion and Flame, 157:1510$1525,2010$.

[20] Christopher Abram, Benoit Fond, Andrew L. Heyes, and Frank Beyrau. Highspeed planar thermometry and velocimetry using thermographic phosphor particles. Applied Physics B, 111(2):155-160, 2013.

[21] C. M. Arndt, J. D. Gounder, W. Meier, and M. Aigner. Auto-ignition and flame stabilization of pulsed methane jets in a hot vitiated coflow studied with highspeed laser and imaging techniques. Applied Physics B, 108(2):407-417, 2012.

[22] B. Bohm, C. Kittler, A. Nauert, and A. Driezler. Diagnostics at high repitition rates: new insights into transient combustion phenomena. In Proceedings of the European Combustion Meeting, 2007.

[23] Isaac Boxx, Christoph Arndt, Campbell Carter, and Wolfgang Meier. Highsped LASER diagnostics for the study of flame dynamics in a lean premixed gas turbine model combustor. In International Symposium on Applications of Laser Techniques to Fluid Mechanics, Lisbon, Portugal, July 5-8 2010.

[24] A Dreizler, S Lindenmaier, U Maas, J Hult, and M Ald. Characterisation of a spark ignition system by planar laser-induced fluorescence of $\mathrm{OH}$ at high repetition rates and comparison with chemical kinetic calculations. Applied Physics B: Lasers and Optics, 70:287-294, 2000. 
[25] C F Kaminski, J Hult, and M Ald. High repetition rate planar laser induced fluorescence of $\mathrm{OH}$ in a turbulent non-premixed flame. Applied Physics B: Lasers and Optics, 68:757-760, 1999.

[26] G. Hartung, J. Hult, R. Balachandran, M. R. Mackley, and C. F. Kaminski. Flame front tracking in turbulent lean premixed flames using stereo PIV and time-sequenced planar LIF of OH. Applied Physics B, 96(4):843-862, 2009.

[27] J Hult, U Meier, W Meier, A Harvey, and C F Kaminski. Experimental analysis of local flame extinction in a turbulent jet diffusion flame by high repetition 2-D laser techniques and multi-scalar measurements. Proceedings of the Combustion Institute, 30(1):701-709, 2005.

[28] W Meier, I Boxx, M Stöhr, and C D Carter. Laser-based investigations in gas turbine model combustors. Experiments in Fluids, 49:865-882, 2010.

[29] Adam M. Steinberg, Isaac Boxx, Michael Stohr, Campbell D. Carter, and Wolfgang Meier. Flow-flame interactions causing acoustically coupled heat release fluctuations in a thermo-acoustically unstable gas turbine model combustor. Combustion and Flame, 157:2250-2266, 2010.

[30] A.M. Steinberg, I. Boxx, C.M. Arndt, J.H. Frank, and W. Meier. Experimental study of flame-hole reignition mechanisms in a turbulent non-premixed jet flame using sustained multi-kHz PIV and crossed-plane OH PLIF. Proceedings of the Combustion Institute, 33(1):1663-1672, 2011.

[31] M. Stöhr, I. Boxx, C. Carter, and W. Meier. Dynamics of lean blowout of a swirlstabilized flame in a gas turbine model combustor. Proceedings of the Combustion Institute, 33(2):2953-2960, 2011.

[32] Michael Stöhr, Isaac Boxx, Campbell D Carter, and Wolfgang Meier. Experimental study of vortex-flame interaction in a gas turbine model combustor. Combustion and Flame, 159(8):2636-2649, 2012.

[33] P.J. Trunk, I. Boxx, C. Heeger, W. Meier, B. Böhm, and a. Dreizler. Premixed flame propagation in turbulent flow by means of stereoscopic PIV and dualplane OH-PLIF at sustained $\mathrm{kHz}$ repetition rates. Proceedings of the Combustion Institute, 34(2):3565-3572, 2013.

[34] Carson D. Slabaugh, Andrew C. Pratt, and Robert P. Lucht. Simultaneous 5 kHz OH-PLIF/PIV for the Study of Turbulent Combustion at Engine Conditions. Applied Physics B: Lasers and Optics, 118(1):109-130, 2014.

[35] Isaac Boxx, Carson D. Slabaugh, Peter Kutne, Robert P. Lucht, and Wolfgang Meier. $3 \mathrm{kHz}$ PIV / OH-PLIF measurements in a gas turbine combustor at elevated pressure. Proceedings of the Combustion Institute, (accepted for publication), 2014.

[36] Carson Slabaugh, Isaac Boxx, Stefanie Werner, Wolfgang Meier, and Robert Lucht. High-Speed Measurements in Partially-Premixed Swirl Flames at Elevated Temperature and Pressure. In AIAA Aerospace Science Meeting, AIAA2015-0670, Kissimmee, Florida, January 5-9 2015.

[37] A Fage and H.C.H Townend. Turbulent Motion Near a Wall. Proc. R. Soc. London Ser. A, 135:656-677, 1932. 
[38] R.J. Adrian and J. Westerweel. Particle Image Velocimetry. Cambridge Aerospace Series. Cambridge University Press, 2010.

[39] D.B. Barker and M.E. Fourney. Measuring Fluid Velocities with Speckle Patters. Opt. Lett., 1(135), 1977.

[40] T.D. Dudderar and P.G. Simpkins. Laser Speckle Photography in a fluid medium. Nature, 270:45-47, 1977.

[41] R. Grousson and K.S. Breuer. Study of Flow Pattern in a fluid by Scattered Laser Light. Applied Optics, 16:2334-2336, 1977.

[42] R J Adrian. Scattering particle characteristics and their effect on pulsed laser measurements of fluid flow: speckle velocimetry vs particle image velocimetry. Applied optics, 23(11):1690-1, June 1984.

[43] Christopher J. D. Pickering and Neil A. Halliwell. Speckle photography in fluid flows: signal recovery with two-step processing. Applied Optics, 23(8):1128, April 1984.

[44] R. Adrian. Particle-Imaging Techniques For Experimental Fluid-Mechanics. Annual Review of Fluid Mechanics, 23(1):261-304, January 1991.

[45] C.E. Willert and M. Gharib. Digital particle image velocimetry. Experiments in Fluids, 10(4), January 1991.

[46] N.T. Clemens and M.G. Mungal. A planar Mie scattering technique for visualizing supersonic mixing flows. Experiments in Fluids, 11(2-3):175-185, 1991.

[47] I. Boxx, C. Heeger, R. Gordon, B. Böhm, a. Dreizler, and W. Meier. On the importance of temporal context in interpretation of flame discontinuities. Combustion and Flame, 156(1):269-271, 2009.

[48] Bruno Coriton, Adam M. Steinberg, and Jonathan H. Frank. High-speed tomographic PIV and OH PLIF measurements in turbulent reactive flows. Experiments in Fluids, 55(6):1-6, 2014.

[49] Bharathram Ganapathisubramani. Investigation of turbulent boundary layer structure using stereoscopic particle image velocimetry. 2004.

[50] A.K. Prasad. Stereoscopic Particle Image Velocimetry. Experiments in Fluids, 29:103-116, 2000.

[51] Christian Willert. Stereoscopic digital particle image velocimetry for application in wind tunnel flows. Measurement Science and Technology, 8(12):1465-1479, December 1999.

[52] O. Özcan, K. E. Meyer, and P. S. Larsen. Measurement of mean rotation and strain-rate tensors by using stereoscopic PIV. Experiments in Fluids, 39(4):771$783,2005$.

[53] a. Lecerf, B. Renou, D. Allano, A. Boukhalfa, and M. Trinit\&\#x000E9;. Stereoscopic PIV: validation and application to an isotropic turbulent flow. Experiments in Fluids, 26(1-2):107-115, 1999. 
[54] M Elsayed, F Scarano, and N G Verhaagen. Particle Image Velocimetry Study of the Flow over a Slender Delta Wing. (June):1-10, 2007.

[55] C.J. Kahler and J. Kompenhans. Multiple plane stereo PIV: Technical realization and fluid-mechanical significance. In 3rd International PIV Workshop, Santa Barbara, 1999.

[56] C.J. Kahler and M. Stanislas. Investigation of wall bounded flows by means of multiple plane stereo PIV. In 10th International Symposium on Applications of Laser Techniques to Fluid Mechanics, Lisbon, Portugal, 2000.

[57] Mathieu Legrand, José Nogueira, Antonio Lecuona, Sara Nauri, and Pedro a. Rodríguez. Atmospheric low swirl burner flow characterization with stereo PIV. Experiments in Fluids, 48(5):901-913, 2010.

[58] P. J. Trunk, I. Boxx, C. Heeger, W. Meier, B. Böhm, and a. Dreizler. Premixed flame propagation in turbulent flow by means of stereoscopic PIV and dualplane OH-PLIF at sustained $\mathrm{kHz}$ repetition rates. Proceedings of the Combustion Institute, 34(2):3565-3572, 2013.

[59] C. T. Smith and C. P. Goyne. Application of Stereoscopic Particle Image Velocimetry to a Dual-Mode Scramjet. Journal of Propulsion and Power, 27(6):1178-1185, 2011.

[60] I. Boxx, C. Heeger, R. Gordon, B. Böhm, M. Aigner, a. Dreizler, and W. Meier. Simultaneous three-component PIV/OH-PLIF measurements of a turbulent lifted, C3H8-argon jet diffusion flame at $1.5 \mathrm{kHz}$ repetition rate. Proceedings of the Combustion Institute, 32 I:905-912, 2009.

[61] A. Stella, G. Guj, J. Kompenhans, M. Raffel, and H. Richard. Applications particle image velocimetry to combusting flows: design considerations and uncertainty assessment. Experiments in Fluids, 30(1):167-180, 2001.

[62] a Melling. Tracer particles and seeding for particle image velocimetry. Measurement Science and Technology, 8(12):1406-1416, 1999.

[63] M. G. MUNGAL, L. M. LOURENCO, and A. KROTHAPALLI. Instantaneous Velocity Measurements in Laminar and Turbulent Premixed Flames Using OnLine PIV. Combustion Science and Technology, 106(4-6):239-265, January 1995.

[64] L Muniz, R.E. Martinez, and Mungal M.G. Application of PIV to turbulent reacting flows. In 8th International Symposium on Applications of Laser Techniques to Fluid Mechanics, pp3.3.1-3.3.8, Libson, Portugal, 1996.

[65] E.F. Hasselbrink, D. Han, M.G. Mungal, and R.K. Hanson. Simultaneous PIV and PLIF measurements in nonpremixed transverse jet flames. In 9th International Conference on Laser Anemometry - Advances and Applications, pp 25.2.125.2.8, Libson, Portugal, 1998.

[66] G. Ingenito, A. Stella, G. Guj, and S. Giammartini. Application of PIV to the analysis of premixed flames. In 9th International Conference on Laser Anemometry - Advances and Applications, pp 3-10, University of Rome, 1998. 
[67] N. Paone. Velocity measurements in turbulent premixed flames: development of a PIV measurement system and comparison with LDV. In 7th International Symposium on Applications of Laser Techniques to Fluid Mechanics, pp15.3.115.3.8, Libson, Portugal, 1994.

[68] D.L. Reuss and M.E. Rosalik. PIV measurements during combustion in a reciprocating internal combustion engine. In 8th International Symposium on Applications of Laser Techniques to Fluid Mechanics, pp37.1.1-37.1.17, Libson, Portugal, 1998.

[69] C. Maurey, A. Cessou, and D. Stepowski. Simultaneous PIV and OH planar LIF in the stabilization region of a lifted turbulent flame. In 9th International Symposium on Applications of Laser Techniques to Fluid Mechanics, pp25.3.125.3.8, Libson, Portugal, 1998.

[70] M. Raffel, C. Willert, and J. Kompenhans. Particle Image Velocimetry - A Practical Guide. Springer, 1998.

[71] LaVision. Lavision flowmaster software manual.

[72] Ulrich Stopper, Manfred Aigner, Wolfgang Meier, Rajesh Sadanandan, Michael Stöhr, and Ik Soo Kim. Flow field and combustion characterization of premixed gas turbine flames by planar laser techniques. Journal of engineering for gas turbines and power, 131(2), 2009.

[73] M Carl, T Behrendt, C Fleing, M Frodermann, J Heinze, C Hassa, U Meier, D Wolff-Gassmann, S Hohmann, and N Zarzalis. Experimental and numerical investigation of a planar combustor sector at realistic operating conditions. Journal of Engineering for Gas Turbines and Power, 123(4):810-816, 2001.

[74] Nikolaos Zarzalis, Thomas Ripplinger, Stefan Hohmann, Matthias Hettel, Klaus Merkle, Wolfgang Leuckel, Göran Klose, Robert Meier, Reiner Koch, Sigmar Wittig, et al. Low-NOx Combustor Development pursued within the scope of the Engine 3E German national research program in a cooperative effort among engine Manufacturer MTU, University of Karlsruhe and DLR German Aerospace Research Center. Aerospace Science and Technology, 6(7):531-544, 2002.

[75] J.C. Broda, S. Seo, R.J. Santoro, G. Shirhattikar, and V. Yang. An experimental study of combustion dynamics of a premixed swirl injector. Symposium (International) on Combustion, 27(2):1849-1856, 1998.

[76] S-Y Lee, S Seo, JC Broda, S Pal, and RJ Santoro. An experimental estimation of mean reaction rate and flame structure during combustion instability in a lean premixed gas turbine combustor. Proceedings of the Combustion Institute, 28(1):775-782, 2000 . 
VITA 


\section{VITA}

Andrew Charles Pratt was born on April 25, 1989 in Fort Wayne, Indiana to Charlie and Roxanne Pratt. He earned his Bachelor of Science in Aeronautical and Astronautical Engineering in 2012. Since then, he has been working on the application of laser based optical measurement systems in high power gas turbine combustion experiments. Andrew is engaged to Frances Williams. 